\title{
Between life and death: reducing phototoxicity in Super-Resolution Microscopy
}

\author{
Kalina L. Tosheva ${ }^{1}$, Yue Yuan ${ }^{1}$, Pedro Matos Pereira ${ }^{2}$, Siân Culley ${ }^{1,3 \otimes^{*}}$, and Ricardo Henriques ${ }^{1,3 \otimes^{*}}$ \\ ${ }^{1}$ MRC Laboratory for Molecular Cell Biology, University College London, London, UK \\ ${ }^{2}$ ITQB-NOVA, Oeiras, Portugal \\ ${ }^{3}$ The Francis Crick Institute, London, UK
}

\begin{abstract}
Super-Resolution Microscopy enables non-invasive, moleculespecific imaging of the internal structure and dynamics of cells with sub-diffraction limit spatial resolution. One of its major limitations is the requirement for high-intensity illumination, generating considerable cellular phototoxicity. This factor considerably limits the capacity for live-cell observations, particularly for extended periods of time. Here, we overview new developments in hardware, software and probe chemistry aiming to reduce phototoxicity. Additionally, we discuss how the choice of biological model and sample environment impacts the capacity for live-cell observations.
\end{abstract}

Phototoxicity | Photodamage | Super-Resolution Microscopy | Fluorescence Correspondence: s.culley@ucl.ac.uk,r.henriques@ucl.ac.uk

\section{Introduction}

The spatial resolution of an imaging system is defined as the capacity to distinguish closely separated features; in light microscopy, this is limited by diffraction to $\sim 200-300 \mathrm{~nm}$. Consequently, microscopy approaches developed to achieve resolutions beyond this limit are termed 'Super-Resolution Microscopy' (SRM) (1). SRM techniques that have recently gained popularity, such as Photoactivated Localisation Microscopy (PALM) (2), Stochastic Optical Reconstruction Microscopy (STORM) (3), Structured Illumination Microscopy (SIM) (4) and Stimulated Emission Depletion (STED) Microscopy (5), have enabled biological discoveries inaccessible to conventional microscopy (6-9). Alongside increased spatial resolution, SRM retains many desirable features of light microscopy techniques, including molecule-specific labelling and the potential for live-cell imaging, unavailable to other high-resolution techniques, such as electron microscopy. However, the live-cell imaging potential of SRM has remained largely untapped as the requirements of most SRM techniques pose various challenges for exploring dynamic processes under physiological conditions. In contrast, such limitations are absent when using fixed specimens.

Resolution increase in SRM is generally achieved at the cost of high-intensity illumination (10). These requirements result in photobleaching, defined as irreversible loss of fluorescence during imaging. However, of greater concern for livecell imaging is phototoxicity. In the context of microscopy, phototoxicity is a broad term encompassing physical and chemical reactions caused by the interaction between light and cellular components, with detrimental effects on the latter $(11,12)$. Correct biological interpretations from live-cell

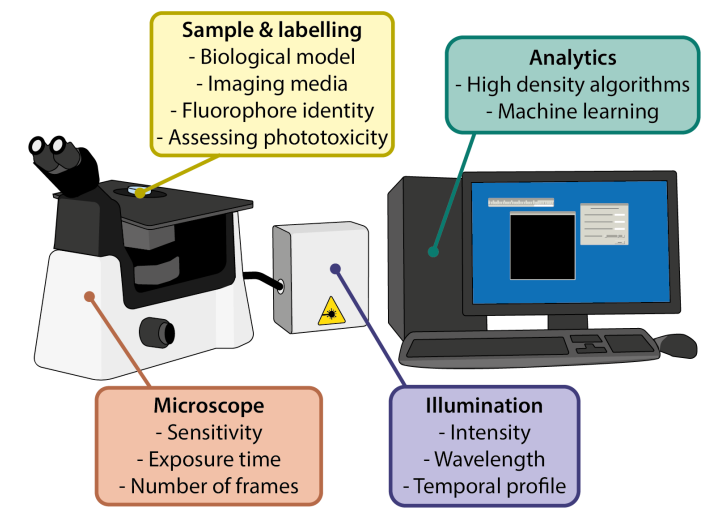

Fig. 1. Summary of the factors that can be optimised to reduce phototoxicity in Super-Resolution Microscopy.

imaging can only be achieved if the observed phenomena progress with minimal perturbation (13).

On a molecular level, the main causes of phototoxicity are photochemical processes that directly damage intracellular components or lead to the production of toxic products within the cell or in its direct environment $(14,15)$. The detrimental effects of ultraviolet (UV) light on cells is particularly well characterised; illumination with UV light can trigger the so-called 'UV-response' (Fig. 2a) (16, 17), DNAstrand breaks $(18,19)$, and thymidine dimerisations (20) (Fig. $2 \mathrm{~b})$, leading to mutations and downstream apoptosis $(21,22)$. Additionally, both UV and visible wavelengths can excite other endogenous photoactive molecules in the cell, such as $\mathrm{NAD}(\mathrm{P}) \mathrm{H}(23)$, flavins $(24,25)$ and porphyrins $(26,27)$. Furthermore, in fluorescence microscopy there are phototoxic effects associated with the fluorescent molecules required for labelling structures $(14,28)$. Upon illumination, both endogenous and exogenous photoactive molecules can be excited to reactive states (most commonly long-lived triplet states) capable of undergoing redox reactions that lead to formation of reactive oxygen species (ROS) (Fig. 2c). ROS are considered the major contributors to phototoxicity $(11,12)$. Their production can occur via direct reaction between the excited molecule and environmental molecular oxygen or via reactions with other neighbouring molecules that generate free radicals (29). ROS have a broad range of negative effects ranging from oxidising proteins, lipids, and DNA, as well as systematic effects such as disrupting the redox homeostasis, signalling pathways and cell cycle $(11,30)$. Notably, ROS production correlates with illumination intensity and photo- 


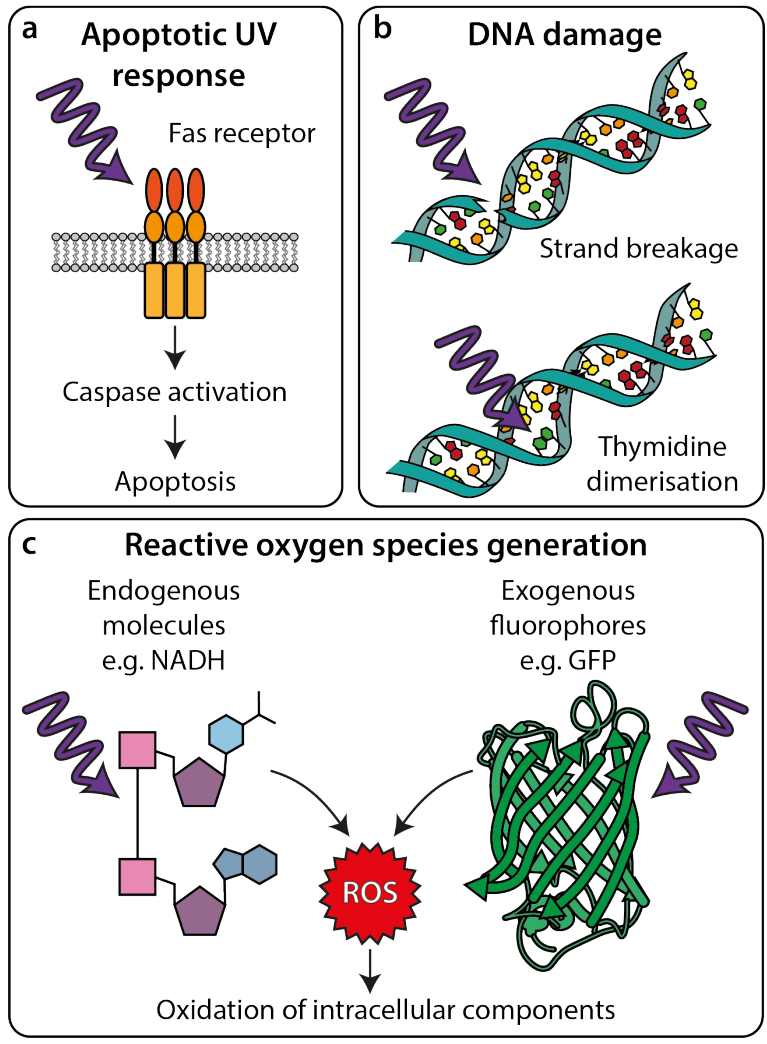

Fig. 2. Interactions of light with cellular components leading to phototoxicity. a UV light can trigger apoptosis by inducing Fas receptor-mediated signalling pathways. b UV light can directly damage DNA by causing strand breakage (top) or thymidine dimerisation (bottom), causing mutations and inducing DNA damage responses. c UV and visible wavelengths can excite photoactive molecules leading to chemical generation of ROS, which can then damage cellular components.

bleaching $(11,14)$. The dependency of SRM on illumination intensities orders of magnitude higher than conventional microscopy $\left(\mathrm{W} / \mathrm{cm}^{2}-\mathrm{GW} / \mathrm{cm}^{2}\right.$ compared to $\mathrm{mW} / \mathrm{cm}^{2}-\mathrm{W} / \mathrm{cm}^{2}$ ) makes these techniques susceptible to phototoxicity $(10,31)$. As a result, there is considerable interest in developing SRM technologies for improved sample health. Here, we will outline the progress in hardware, software and probe development as well as choices in biological model and sample preparation that can help improve live-cell SRM (Fig. 1).

\section{Quantifying phototoxicity in microscopy}

Measuring phototoxicity in microscopy is not a trivial problem, as evidenced by the sparsity of the available literature $(11,12)$. This is not entirely surprising, as phototoxicity is mediated by many factors (Fig. 1). These include illumination wavelength, intensity and duration of illumination, the illumination regime (e.g. LED illumination vs. laser illumination, laser-scanning vs. light-sheet), and the number of imaged 3D-planes (33-38). Additionally, illumination tolerance can vary substantially between specimens (see Biological models and sample preparation section), and experimental stress can influence a specimen's sensitivity to illumination (13). For example, a procedure as routine as transfection or the addition of a drug has been shown to dramatically increase cellular sensitivity to light $(10,39)$.

How does one approach a problem as versatile as measur- ing phototoxicity? An intuitive and common way of assessing photodamage is by measuring photobleaching (40-43). However, phototoxicity and photobleaching are two separate processes; while toxic ROS are produced during photobleaching, they can also be generated independently of this process $(14,44)$. Therefore, phototoxicity can commence prior to a detectable reduction in fluorescence, making photobleaching an unreliable read-out for photodamage in the context of live-cell imaging (11). More importantly, photobleaching rates give no information on the health and viability of the specimen. Thus, a better phototoxicity measure would have a read-out related to the properties of the sample itself, rather than the properties of the fluorescence (35).

There are several in vitro assays for post-imaging assessment of the health and viability of a specimen that can be used to indicate whether phototoxicity occurred (Fig. 3a). These include detection of toxic ROS, fragmentation and oxidation of DNA strands, reduced metabolic activity, loss of membrane integrity and the expression of stress- and apoptosis-related proteins (45-50). The advantages are that these assays provide an inexpensive and simple specimen viability evaluation. Thus, different illumination conditions can be tested and viability can be assessed each time. However, for such assays the measurement is limited to a single timepoint and imaging cannot be recommenced after performing the assay.

A more dynamic and practical approach entails monitoring changes in relevant biological parameters during imaging (Fig. 3b, c). Cellular processes particularly photosensitive (i.e. rapidly perturbed by light) are excellent read-outs. For example, a commonly employed method is measuring changes in cytosolic calcium concentration using calciumsensitive fluorescent probes (50-53) (Fig. 3b, top). This strategy was used to evaluate live-cell STED microscopy by monitoring differences in intracellular calcium concentration between control cells and STED-imaged cells. This method showed that while there is little difference between calcium concentration in control and STED-imaged cells when using excitation and STED-lasers with wavelengths $>600 \mathrm{~nm}$, responses indicative of cell damage were observed with shorter illumination wavelengths and when longer STED-laser dwell times were used (28). Other processes exist that make suitable read-outs for phototoxicity, including changes in mitochondrial membrane potential $(32,41)$ (Fig. 3b, bottom), reduction of chromosome movement (54), and slowing of microtubule growth (10). It is worth highlighting that, regardless of the process chosen, care must be taken when employing fuorescent probes for visualising these read-outs $(46,55)$.

There are image-based phototoxicity measurements that can be performed without fluorescent labels. These often rely on identifying changes in cell morphology indicative of entry into apoptosis, such as blebbing or cell rounding $(10,13$, 32,56 ), for example by using transmitted light imaging (Fig. $3 \mathrm{c})$. This approach was recently used to train a deep convolutional neural network, referred to as 'DeadNet', with the objective to automate phototoxicity detection and quantification from transmitted light images (57). However, despite widespread use, relying on morphology as a read-out has two 


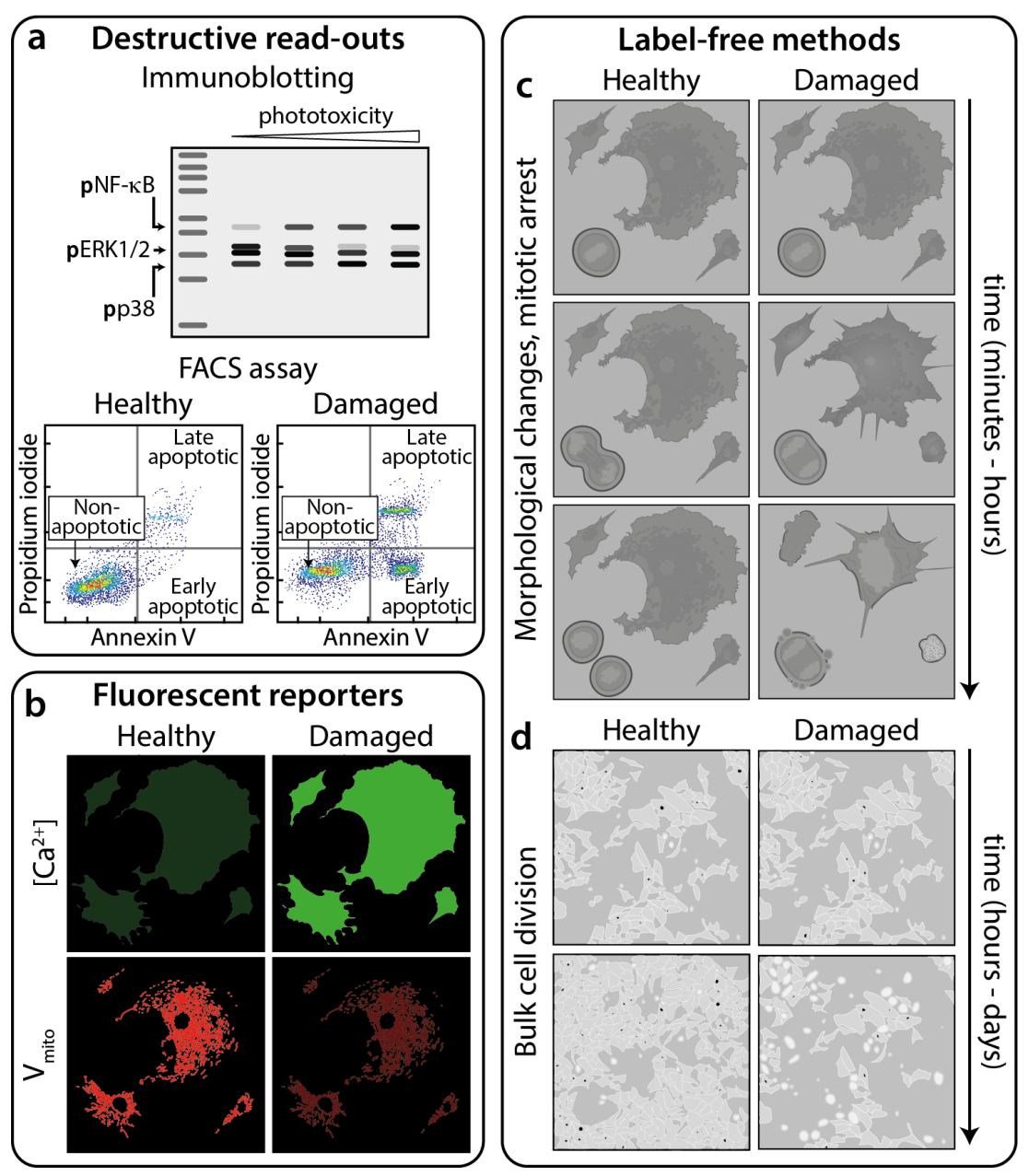

Fig. 3. Methods for measuring phototoxicity. a 'Destructive read-outs' are techniques prohibiting further imaging of the sample. These include blotting for phosphorylated forms of proteins present in damage-activated pathways (32) and flow cytometry for determining the population of cells expressing, for example, apoptotic markers such as annexin V. b 'Fluorescent reporters' are additional indicators added to the sample during imaging whose fluorescence signal changes in response to e.g. intracellular $\mathrm{Ca}^{2+}$ concentration (top) or mitochondrial membrane potential (bottom). 'Label-free methods' of quantifying phototoxicity involve: c short-term observation of cell division and morphology and $\mathbf{d}$ proliferation of cells in culture following imaging. limitations: first, even experienced researchers can struggle to identify subtle changes in morphology, thus biasing the results (e.g. by annotating ambiguous cases incorrectly (57)); second, when changes become obvious, they usually represent an extreme phenotype indicative of irreversible damage. Thus, they cannot account for early damage that may arise even as cells display a healthy morphology $(12,58)$.

In this context, a read-out that deserves special mention is cell division (Fig. 3c, d): a well-characterised biological process with easily identifiable phases. It is highly regulated and sensitive to various perturbations, including illumination and changes in ROS concentrations $(14,30)$. This makes cell cycle an excellent read-out for detection and quantification of phototoxicity (58), with both continuous (Fig. 3c) and endpoint (Fig. 3d) measurements possible. Delay in mitotic progression has been used successfully to detect perturbations in the health of both cultured cells and developing embryos (33-36). Additionally, evaluating colony formation or number of cell divisions after illumination (typically assessed after a period of one or more cell cycles) can be indicative of long-lasting damage $(11,28)$ (Fig. 3d). This approach was used to perform extensive characterisation of photodamage under illumination conditions commonly used in singlemolecule localisation microscopy (SMLM) (10). The viability of several different cell lines was determined 20-24 h post illumination, a strong correlation between shorter illumination wavelengths and increased cell death was shown, particularly at high intensities. However, results also suggested that long-term cell viability is possible even with illumination wavelengths as short as $405 \mathrm{~nm}$, provided the integrated light dose is small, preferably with continuous rather than pulsed illumination. Naturally, a limitation exists in employing these methods to assess phototoxicity in post-mitotic systems, e.g. primary neuron cultures. However, for relevant models, choosing mitosis as a read-out has the significant advantage of allowing phototoxicity assessment based on label-free transmitted light images $(10,28,34)$, minimising the introducing additional damage during evaluation.

From reports of phototoxicity in literature, several conclusions can be drawn to guide live-cell friendly SRM. Firstly, red-shifted wavelengths are preferable to shorter wavelengths. In particular, UV wavelengths should be avoided wherever possible $(10,28,34)$. Furthermore, several studies demonstrate that lower intensity illumination with longer exposure is less damaging than short intense bursts or pulses of illumination $(10,35,40)$. Most importantly, a recurrent message throughout the literature is that higher illumination intensities are more damaging than corresponding imaging conditions with lower illumination intensities. We anticipate that real-time phototoxicity measurements will become com- 

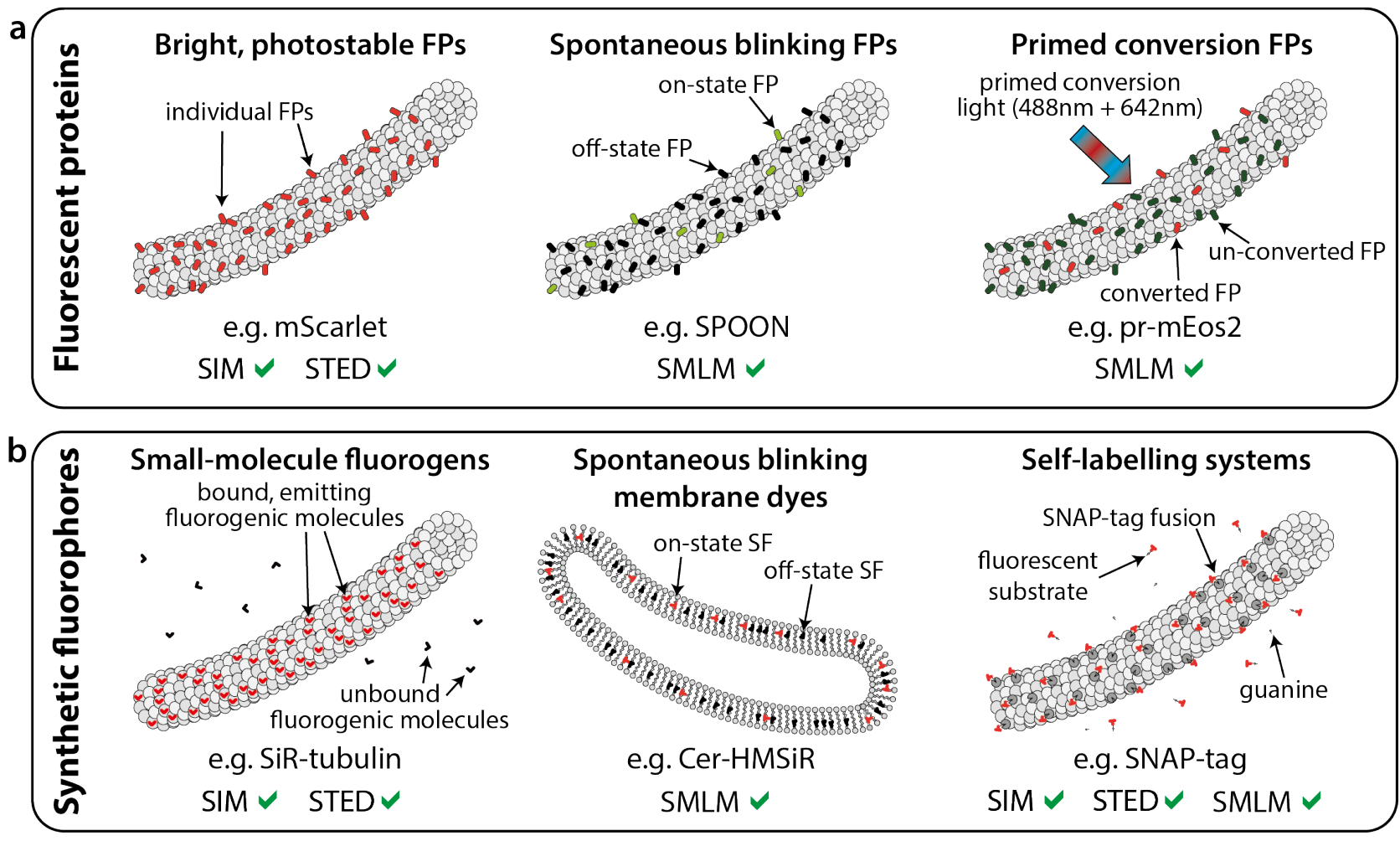

Fig. 4. Low phototoxicity fluorescent probes and labelling for live-cell Super-Resolution Microscopy. Various recently-developed fluorescent protein- (a) and synthetic fluorophore- (b) based methods for labelling in live-cell super-resolution. All labels are shown attached to a microtubule as an example of an intracellular structure, with the exception of the Cer-HMSiR membrane dye in $\mathbf{b}$.

monplace in both diffraction-limited microscopy and SRM, and that future SRM techniques will be accompanied by a thorough description of how they impact living samples. Concomitantly, for SRM users, awareness of strategies for minimising phototoxicity is crucial.

\section{Fluorescent probe development for live-cell Super-Resolution Microscopy}

SRM techniques have distinct requirements for fluorescent probes. SIM quality relies on collecting images of high Signal-to-Noise Ratio (SNR), generally achieved by labelling with fluorophores of high brightness and resistance to photobleaching. In STED, fluorophores must not only be bright but also possess a large Stokes-shift and stimulated emission cross-section at the STED wavelength (59). SMLM techniques have the most demanding labelling requirements - fluorophores must be capable of cycling between 'on' and 'off' states with appropriate kinetics, a high quantum yield in the on-state, and a very low quantum yield in the off-state.

Several fluorophores and probes have been developed specifically for SRM $(60,61)$. However, while many specialised fluorophores exist for fixed specimens (62), there are far fewer options available for live-cell imaging. An inappropriate choice of fluorophore for live-cell SRM will not only lead to low quality images downstream (63), but also inevitably impact acquisition settings and hence phototoxicity $(10,64)$. As for most fluorescence microscopy techniques, the two classes of fluorophores used in SRM are fluorescent proteins (FPs) (Fig. 4a) and synthetic fluorophores (SFs) (Fig. 4b).
FPs are the usual choice for live-cell imaging as they can be fused to a target of interest via genetic encoding, but at the cost of reduced brightness compared to SFs. The recent development of bright and photobleaching-resistant FPs has expanded the options for SIM and STED (Fig. 4a, left). Examples of these new FPs are mNeonGreen $\left(\lambda_{\text {ex }}=506 \mathrm{~nm}\right)$ (65), mScarlet $\left(\lambda_{\text {ex }}=569 \mathrm{~nm}\right)(66)$ and $m G a r n e t\left(\lambda_{\text {ex }}=598 \mathrm{~nm}\right)$ (67). SMLM techniques generally require photoswitchable fluorophores (e.g. mEos3.2, rsKame) $(68,69)$. Despite the availability of several photoswitchable FPs, their use in livecell imaging remains challenging $(10,64)$. The chief reason is that transitions between off- and on-states are typically modulated by UV illumination. The combination of this with high intensity excitation for detection of molecular positions results in a short window for live-cell SMLM studies. To reduce phototoxicity in SMLM, FPs that do not require UV pumping for photoswitching are being developed (Fig. 4a, centre), with one such example being SPOON (70). Primed conversion is another promising UV-independent approach to induce photoswitching (71) (Fig. 4a, right). Thereby a combination of blue and near-infrared illumination induces photoconversion in Dendra2 and the newly developed primedconversion protein pr-mEos2 $(71,72)$. Recently, a general mechanism for primed conversion was described, which is anticipated to accelerate the development of more FPs that can be photoconverted with this live-cell friendly approach (73). FPs for other specific SRM techniques have also been developed (e.g. Skylan-NS for non-linear SIM or GMars for REversible Saturable/switchable OpticaL Fluorescence Transitions, RESOLFT) $(74,75)$. 
The second alternative, SFs (Fig. 4b), are small chemically synthesised probes. These have higher quantum yields and are more robust against photobleaching than FPs (76-79). While there are some cell-permeable SFs that can be used to label specific proteins (e.g. fluorogens such as $\mathrm{SiR}$-tubulin and SiR-actin) (Fig. 4b, left) $(80,81)$ or cell compartments directly (e.g. Membright, ER-Tracker or MitoTracker) (Fig. $4 \mathrm{~b}$, centre) $(77,82-84)$, additional 'linker' molecules are normally required to associate SFs with the structure of interest. These linkers must bind the target structure with high affinity and specificity (e.g. antibodies and DNA/RNA scaffolds, usually using amine- or thiol-reactive derivatives of the SF) (85). However, many of these high-affinity linkers and SFs are not cell-permeable, which limits their use in live-cell SRM to labelling of cell-surface molecules. If genetic encoding is possible and preferable, cell-permeable SFs can be combined with flexible self-labelling systems, such as SNAP-tag, Halo-tag or FlAsH (Fig. 4b right) (86-89). An elegant example of such an approach is the use of Cox8ASNAP fusion labelled with SNAP-Cell SiR for STED. This has enabled the visualisation of the dynamics of mitochondrial cristae with $\sim 70 \mathrm{~nm}$ resolution (90).

SFs have also been engineered for live-cell SRM. Spontaneously blinking synthetic fluorophores (e.g. HMSiR) have been recently developed (Fig. 4b, center). They do not require UV irradiation or cytotoxic additives (such as thiol) to induce photoswitching $(91,92)$. High photostability SFs have also been developed, enabling live-cell STED (79, 93-95).

A final regime for live-cell SRM-compatible labelling is based on site-specific conjugation of fluorophores to a target of interest, through genetic code modifications and click chemistry (96-98) (Fig. 4b, right). These approaches combine the benefits of site-specific labelling (as is the case for FPs) with no requirement for protein expression and bright labels (as is the case for SFs).

\section{Biological models and sample preparation}

Care should be taken when selecting a biological model for SRM. Cellular sensitivity to light exposure can vary based on cell type and species $(10,13,45)$, and in the case of whole organisms, developmental stage $(12,35)$. Phototoxicity has been documented for different cell types, ranging from primary cells $(12,45)$ to various immortalised cell lines $(10,25,39,99)$. One such study focuses on immortalised cell lines, where it shows that COS-7 and U2OS cells exhibit similar photosensitivity, whereas HeLa cells are substantially more robust, potentially making the latter a more suitable system for live-cell SRM studies (10). Another study illustrated the effect of photodamage on primary cells from rat central nervous system (45). Here, illumination with blue light could induce morphological changes, differentiation or cell death depending on the cell type.

When imaging whole organisms, earlier developmental stages from the same species tend to be more photosensitive than later (11). Furthermore, different model organisms display variable photosensitivity. For example, fruit fly embryos and nematode worms have higher illumination tolerances than zebrafish embryos, corals or cultured cells $(12,13)$. Even within the same cell, different intracellular structures exhibit different responses to illumination $(28,100)$.

Photodamage can be mitigated through additional sample preparation steps. Established strategies centre on preventing photobleaching by modifying the sample environment. As photobleaching can contribute to phototoxicity via ROS production (44), strategies to reduce photobleaching could also help ameliorate phototoxicity $(14,28,101)$. One strategy is to modify the environmental conditions prior to or during imaging. A prime example is removal of oxygen, the main effector of photobleaching (102), from the culture medium. This can be achieved by bubbling nitrogen through the medium during imaging. This yields an increased photostability $(103,104)$ and, since oxygen is directly involved in the production of ROS, also reduces light-dependent oxidative stress on the sample. It has also been shown that growing cells in a hypoxic environment (3\% oxygen) yielded a $25 \%$ increase in mitosis entry after blue light irradiation (34). Other approaches to reduce oxygen in the medium involve the addition of commercially available oxygen-scavengers such as the Oxyrase ${ }^{\circledR}$ enzyme complex (developed by Oxyrase, Inc., Mansfield, Ohio). In combination with suitable substrates, such as D/L-lactate or D/L-succinate, these enzymes catalytically reduce the concentration of oxygen and free radicals present in the medium, thus minimising photobleaching and phototoxicity $(105,106)$. While these approaches could improve live-cell SRM, it should be noted that they are only suitable for specimens which can tolerate hypoxia or anoxia. Notably, some fluorophores used in SRM require oxygen scavenger systems to photoswitch, however, these buffers typically use cytotoxic compounds such as thiols, making them unsuitable for live-cell imaging.

A different strategy for reduction of ROS during imaging involves supplementing the media with antioxidants. Antioxidants are molecules that prevent oxidation in a biological context (107). Among antioxidants, Trolox, the soluble form of vitamin $\mathrm{E}$, has been shown to have a protective effect for a number of cell lines due to its ROS-neutralising properties (108). The presence of the antioxidant in the sample medium has been shown to increase the number of postillumination mitotic cells by up to $38 \%$ compared to cells illuminated without Trolox (34). However, this molecule is not suitable for SMLM, as it has been shown to inhibit fluorophore blinking (109). Another antioxidant used in microscopy is rutin, a plant flavonoid shown to reduce EGFP reddening $(110,111)$, although no direct reduction of phototoxicity was demonstrated. A notable example of a medium additive for live-cell imaging is the vitamin- and antioxidantrich 'Supplements for Optogenetic Survival' (SOS). SOS has been shown to increase viability and reduce photodamage in several cell types of the rat central nervous system (45).

There are chemicals used in mounting media, such as various antioxidants, triplet-state quenchers and radical scavengers, that can be used for photobleaching reduction and ROS neutralisation. These include ascorbic acid (112), npropyl gallate (112-114), p-phenylenediamine (114-116), 

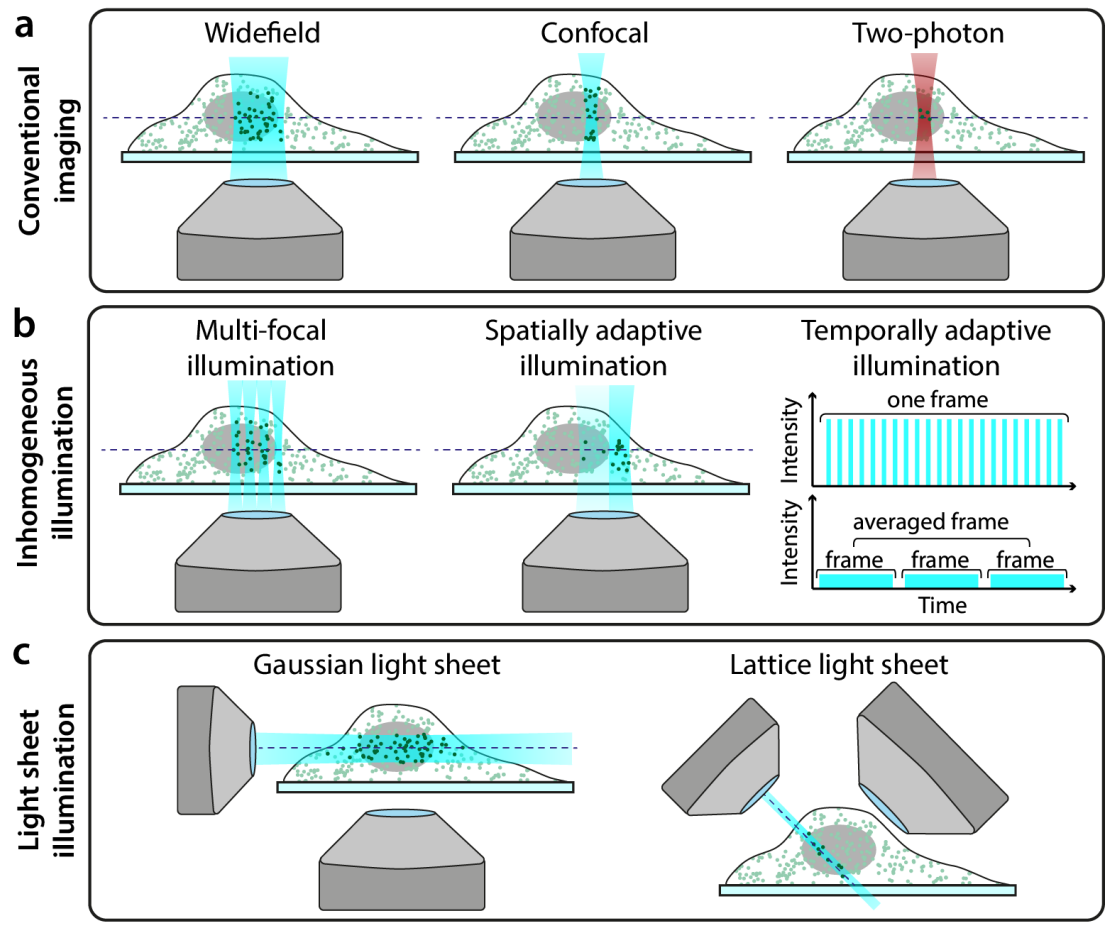

Fig. 5. Hardware modalities for conventional and low-phototoxicity Super-Resolution Microscopy. a Microscopy illumination regimes for conventional fluorescence imaging. b Examples of regimes that reduce light dose to the sample by inhomogeneous illumination. c Examples of light-sheet microscopy geometries. 1,4-diazobicyclo(2,2,2)-octane (DABCO) (114, 117), mercaptoethylamine (MEA) and cyclooctatetraene (COT) (112). Their presence in mounting media for reduction of photobleaching is well characterised $(112,115,118)$, however there is no comprehensive study on the use of these chemicals in live-cell imaging. As a result, there is little information regarding biocompatible working concentrations or biological side effects. Therefore, while potentially useful, they require further exploration prior to use in live-cell SRM.

Some substances commonly used as supplements are known also to cause phototoxicity, such as molecules with benzene rings which are intrinsically fluorescent (111). For example, common cell media components, such as riboflavin and pyridoxal, can enhance oxidative reddening of GFPs; this effect accounts for a considerable part of GFP photobleaching (119). Depleting these substances increases GFP photostability, indirectly reducing photodamage (110). Additionally, the combination of riboflavin and tryptophan in media generates ROS and induces cytotoxicity upon illumination, whereas their removal alleviates this effect $(120,121)$. Finally, the study that established the SOS supplement (45) used it in combination with the photoinert media NEUMO and MEMO, which also lack riboflavin. These media were specifically developed to prevent phototoxicity of nervous system cells. A confounding example is 4-(2-hydroxyethyl)1-piperazineethanesulfonic acid (HEPES), commonly used as a replacement for carbon dioxide buffering during imaging (58). However, early reports demonstrated that HEPESbuffered media exposed to low-intensity white light can generate toxic hydrogen peroxide with detrimental effects on thymocyte or T-cell culture $(122,123)$.

There is still a lack of information on SRM sample preparation reducing phototoxicity. Many principles can be trans- ferred from conventional fluorescence imaging. These include assessing photosensitivity of the biological model, environmental conditions, and attention to media composition.

\section{Hardware developments for improved live- cell imaging}

The microscope configuration has a substantial impact on the amount of photodamage experienced by a specimen. Fig. 5a shows the common illumination regimes for conventional microscopy and SRM (widefield for SIM and SMLM, confocal for STED). Basic optimisations of the microscope body, for example minimising photon loss in the detection path by using high-quality filters and sensitive detectors, will reduce the illumination burden to achieve suitable SNR (101). In SRM approaches, microscopes are built with high-quality components, often having bespoke solutions to maximize signal detection $(124,125)$. In addition, the ever-present phototoxic high-intensity illumination requirements of most SRM techniques can be further ameliorated using dedicated hardware designs. Interestingly, a recent study shown low-illumination live-cell SRM immediately followed by in situ fixation of the sample and high-illumination SRM (126). This approach combines the collection of temporal information in livingcells with a mild resolution increase, then capture of higher resolution for a specific timepoint upon fixation.

In the case of STED, the presence of a second high-intensity laser beam (depletion laser) in addition to a confocal excitation beam confers the high phototoxicity of this method. However, the properties of both beams can have a substantial impact on sample photodamage. It has been shown in confocal microscopy that nanosecond pulsed, rather than continuous, excitation can reduce photobleaching, and that averaging multiple fast scans is less phototoxic than acquiring 
a single slow scan (41) (Fig. 5b, 'Temporally adaptive illumination'). The properties of the excitation beam have also been explored specifically in STED. For example, reducing the pulsing rate of the excitation laser allows time for longlived triplet states to relax which leads to decreased photobleaching (127). Similarly to confocal microscopy, scanning at a higher rate in STED has been shown to reduce photobleaching (42); this is enabled by using fast resonant scanning mirrors rather than slower galvanometer scanning mirrors to scan the beam pair through the sample. Another method described reducing phototoxicity in STED is by using two-photon excitation (Fig. 5a, 'Two-photon'). As twophoton excitation only excites fluorophores within the focal volume of the beam (rather than along the entire beam path, as is the case in single-photon excitation), it is often considered a more live-cell friendly imaging regime $(53,128)$. Indeed, live-cell STED has been successfully demonstrated with two-photon excitation $(129,130)$ although while the former paper claims that there is no photodamage to the sample, this is not quantified. It should be noted that two-photon excitation does however increase local heating, which can damage the sample in a non-fluorophore mediated manner (131). Using a continuous wave $(\mathrm{CW})$ laser for the depletion beam, rather than a fast pulsed laser, can also help reduce phototoxicity through the gSTED (gated-STED) approach (132). In this method, rather than continually increasing the intensity of the depletion beam to increase resolution (as is the case for most preceding STED techniques), time-gated detectors are used to reject fluorescence detected immediately after excitation. This signal is contaminated by emission that should have been suppressed by the depletion beam; when present, these photons erode the resolution of the final image. Thus, gSTED allows for a STED resolution improvement, independent of increasing light dose to the sample (133).

SIM is generally considered the least phototoxic SRM technique (134). However, it still requires the acquisition of several frames (often $\geq 9$ ) at high SNR in order to generate the final reconstructed image. Several approaches have been developed to reduce the number of frames required for a SIM reconstruction, including pixel reassignment and image scanning microscopy (ISM) methods. One example is multifocal structured illumination microscopy (MSIM, (135)), which combines principles from SIM and confocal microscopy to scan an array of spots across the sample for fast live-cell imaging with resolution doubling (Fig. 5b, 'Multi-focal illumination'). Another method, rapid nonlinear ISM (136), combines ISM with two-photon excitation and second-harmonic generation for low phototoxicity imaging. A wide range of such SIM-based techniques exist, and have been rigorously compared elsewhere $(134,137)$. It has been demonstrated recently that using sub-millisecond pulses as excitation in SIM (when combined with novel analytics as described below) reduced photobleaching and enables longterm live-cell imaging (138).

For widefield microscopy in general, light-sheet techniques are rapidly becoming a staple for live-cell imaging. Lightsheet microscopy involves illuminating the sample with a thin sheet of light and then detecting the fluorescence perpendicular to the direction of sheet propagation (139, 140) (Fig. 5c, 'Gaussian light sheet'). This confers low phototoxicity as only the part of the sample being imaged is illuminated without the need for non-linear optical processes (which is the case in two-photon microscopy). Indeed, light-sheet microscopy was named the Nature Methods technique of the year in 2014, in part due to its low phototoxicity (141). There are several ways in which light-sheet microscopy schemes can yield super-resolution with reduced phototoxicity. Superresolution in live samples has been demonstrated using lightsheet microscopy by simply combining this illumination geometry with SRM techniques such as SMLM (142-144) and RESOLFT (145). However, the employed SRM methods still require high-intensity illumination, and thus such composite techniques do not exploit the inherent low phototoxicity of light-sheet imaging. Therefore, a more elegant approach involves illuminating the sample with a light-sheet regime followed by the application of SMLM analytics designed for ultra-high-density datasets, which allows for reduction of the illumination power ((146) and Analytics section, see below). The more widely-explored method for combining SRM and light-sheet microscopy has been the use of novel methods for generating and shaping the light-sheet. Bessel beams have been used to generate thinner light-sheets (147), and these beams have also been extended to incorporate SIM (148). The latter strategy has also been demonstrated on a system with two counterpropagating light-sheets formed using standard Gaussian beams (149). The most radical and live-imaging-friendly light-sheet SRM technique developed to date is lattice light-sheet microscopy (150) (Fig. 5c, 'Lattice light sheet'). This has demonstrated 3D time-lapse superresolution imaging in both cultured cells and intact model organisms with minimal phototoxicity.

An interesting approach to reducing the illumination dose in SRM is using spatially varying illumination depending on the structural content of the imaging region (Fig. 5b, 'Spatially adaptive illumination'). This approach was originally demonstrated for confocal imaging (48) and has since been extended to SIM (151), RESOLFT (152) and indeed lightsheet microscopy (141). There is also a range of adaptive illumination STED techniques that have been developed (153155), and while these predominantly focus on reducing light dose in the context of photobleaching, this will concomitantly also impact the live-cell compatibility of these techniques.

\section{Analytical approaches to live-cell Super- Resolution Microscopy}

Analytics can be used to extract super-resolution information from images acquired at low illumination, and thus low phototoxicity (Fig. 6). Such techniques are generally based on SMLM principles, but improve its live-cell compatibility (Fig. 6a). In SMLM, when high intensity illumination is used, fluorophore blinking is sparse and thus the wellseparated single molecules are straightforward to detect and localise with high accuracy and precision $(156,157)$. However, as intensity is decreased towards a lower phototoxic- 


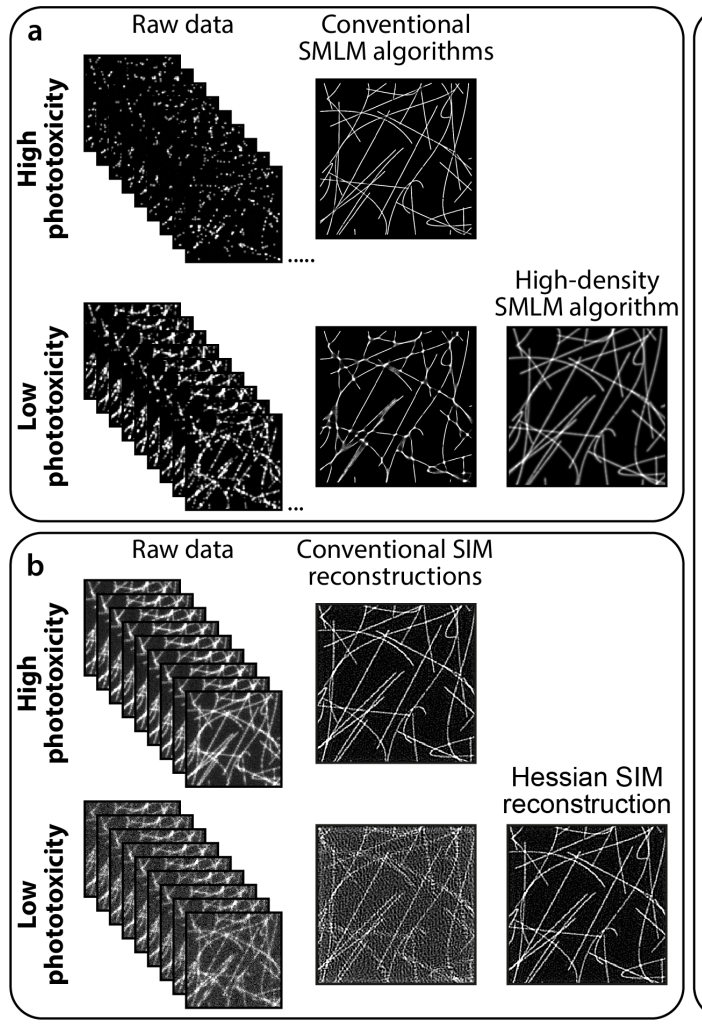

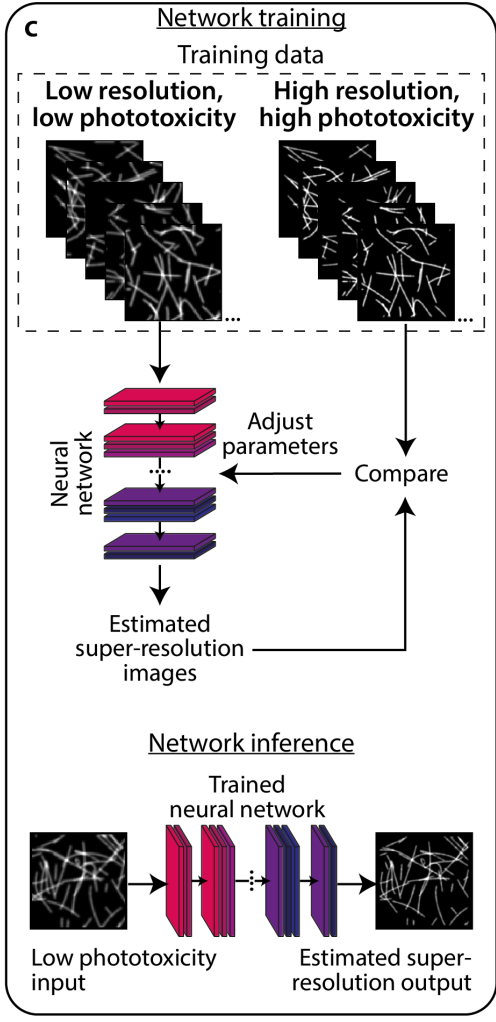

Fig. 6. Analytics to complement lowphototoxicity imaging regimes a Top: typical SMLM images are successfully reconstructed from sparse blinking raw data acquired under high phototoxic illumination. Bottom: reducing phototoxic illumination leads to more emitting fluorophores per raw data frame. When reconstructed using conventional SMLM algorithms, these produce low-quality images containing artefacts. High density SMLM algorithms can produce better quality images from such datasets. b Top: typical SIM imaging involves acquiring 9-25 raw images (depending on the number of grating rotations and phases) at high SNR, which can be successfully reconstructed using conventional SIM algorithms. Bottom: decreasing the illumination intensity, and thus SNR of the raw images, leads to artefacts in images reconstructed using conventional methods. The Hessian SIM deconvolution algorithm can bypass this limitation (138). c Deep neural networks can be trained to infer super-resolution information from e.g. low-resolution diffraction-limited or low-quality super-resolution images. In this example, a neural network can be trained on pairs of low resolution/super-resolution images of the trained structure ('Network training'). The trained network can then be applied to unseen low resolution images to infer the super-resolution equivalents ('Network inference'). ity regime, blinking becomes more dense and molecules become increasingly overlapped. Such datasets require specialised algorithms to extract molecule locations. The first example of such an algorithm was Super-Resolution Optical Fluctuation imaging (SOFI), where the temporal statistics of fluorophore intensity oscillations are used to generate images with sub-diffraction resolution (158). Indeed, SOFI has been used to image live cells (159) although only for short periods of time due to the requirement for UV illumination to induce photoswitching. Another algorithm developed for analysing datasets with dense blinking is 3B (160), where super-resolution images can be obtained from datasets imaged with a xenon arc lamp rather than lasers. However, both SOFI and 3B techniques still rely on photoswitchable fluorophores, which have drawbacks discussed above. The Super-Resolution Radial Fluctuations (SRRF) algorithm allows for the reconstruction of super-resolution images from datasets containing non-photoswitchable fluorophores such as GFP $(161,162)$. SRRF has been shown to work on datasets obtained with confocal and LED-illuminated microscopes, with the latter enabling continuous live-cell imaging for $>30$ minutes (163). However, SRRF cannot retrieve resolutions in these regimes as high as those achievable with photoswitchable fluorophores.

While most analytical developments for live-cell SRM centre on SMLM-based techniques, there are also analytics for enabling lower phototoxicity imaging in SIM and STED. Hessian-SIM is a deconvolution algorithm that can obtain high-quality SIM images from raw data acquired at low signal-to-noise ratio (138) (Fig. 6b). This overcomes a substantial barrier in SIM, in that conventional SIM reconstruc- tion algorithms perform poorly on low-illumination datasets, leading to artefacts within the resulting images. Approaches have also been proposed for low-power STED microscopy based on reconstructing images with knowledge of fluorescence lifetime changes induced by the STED beam $(75,164)$.

A rapidly evolving field in microscopy image analysis is the use of machine learning (ML)-based techniques $(165,166)$. Such techniques are used for diverse applications including object segmentation, denoising, and structure prediction, and these can also be extended to SRM (Fig. 6c). One example is Content Aware Image Restoration (CARE), where a neural network is trained on high illumination intensity datasets (i.e. high phototoxicity) and used to denoise corresponding datasets acquired at much lower illumination intensities (167). CARE was demonstrated to enhance resolution of GFP-tagged microtubules to a similar extent to SRRF analysis of the same data, but with higher quality and higher temporal resolution. There are also specialised ML algorithms for super-resolution applications. ANNA-PALM is a method that, after training a neural network with sparse SMLM data, can reconstruct super-resolution images from dense data and a correspondingly lower number of frames (168). While not demonstrated in live-cell data, this technique could in theory alleviate phototoxicity with minimal sacrifice to spatial resolution by imaging photoswitchable FPs with lower illumination intensity. Other ML-based techniques have also allowed for prediction of enhanced resolution images from low illumination diffraction-limited images (Fig. 6c), for example: converting confocal to Airyscan-type or STED-type images $(75,169)$; or widefield to SIM-type images $(75)$. 


\section{Discussion and outlook}

High quality live-cell fluorescence microscopy involves compromising between four key properties: SNR, imaging speed, spatial resolution, and sample health (11). We present an overview of the challenges faced on how to balance the latter two properties in live-cell SRM, highlighting potential strategies to maximise resolution while minimising phototoxicity. As commercial super-resolution systems become commonplace in biological labs and open-source microscope hardware becomes more widespread, there is a growing desire to translate cell biology experiments from conventional diffraction-limited microscopes to higher resolution alternatives. However, the cost of this increased resolution is often the sample health. Users must be aware of what phototoxicity is, how to detect it, and methods that can be used to ameliorate it. Unfortunately, there are very few dedicated studies discussing phototoxicity specifically in $\operatorname{SRM}(10,28)$.

It is clear that there are several frontiers for optimising SRM protocols for minimising phototoxicity, and a much-needed development in the field is a non-perturbing robust indicator of sample health during imaging. There is already software available for assessing the quality and resolution of SRM images $(170,171)$, and if there were comparative analytics for phototoxicity this would provide a numerical framework for experiment optimisation.

As super-resolution microscopes become increasingly standard equipment in biological research, users must be aware of their limitations in live-cell imaging. Many of the suggestions offered in this review for reducing phototoxicity remain under active development, and it is imperative for users to follow progress in hardware, analytics and fluorophores to ensure that they are minimising photodamage to samples.

\section{ACKNOWLEDGEMENTS}

This work was funded by grants from the UK Biotechnology and Biological Sciences Research Council (BB/R000697/1; BB/R021805/1; BB/S507532/1) (R.H.), the UK Medical Research Council (MR/K015826/1) (R.H.), the Wellcome Trust (203276/Z/16/Z) (S.C., R.H). K.L.T. supported by a 4-year MRC Research Studentship and Y.Y. by a 4-year BBSRC Research Studentship. We would like to thank Dr. David Albrecht (Max Planck Institute for the Science of Light, Erlangen, Germany), Dr. Agathe Chaigne (UCL, London, UK), Dr. Gautam Dey (UCL, London, UK), Dr. Caron Jacobs (University of Cape Town, Cape Town, South Africa) Ms. Megan Jones (UCL, London, UK), Dr. Christophe Leterrier (Aix Marseille Université, Marseille, France), Dr. Apostolos Papandreou (UCL, London, UK) and Dr. Uwe Schmidt (Max Planck Institute of Molecular Cell Biology and Genetics, Dresden, Germany) for valuable advice and feedback.

\section{Bibliography}

1. Lothar Schermelleh, Alexia Ferrand, Thomas Huser, Christian Eggeling, Markus Sauer Oliver Biehlmaier, and Gregor PC Drummen. Super-resolution microscopy demystified. Nature Cell Biology, 21(1):72, 2019. doi: 10.1038/s41556-018-0251-8.

2. Eric Betzig, George H Patterson, Rachid Sougrat, O Wolf Lindwasser, Scott Olenych, Juan S Bonifacino, Michael W Davidson, Jennifer Lippincott-Schwartz, and Harald F Hess. Imaging intracellular fluorescent proteins at nanometer resolution. Science, 313(5793): 1642-5, 2006. doi: 10.1126/science. 1127344 .

3. Michael J Rust, Mark Bates, and Xiaowei Zhuang. Sub-diffraction-limit imaging by stochastic optical reconstruction microscopy (STORM). Nature Methods, 3(10):793, 2006. doi: $10.1038 /$ nmeth 929.

4. Mats GL Gustafsson. Surpassing the lateral resolution limit by a factor of two using structured illumination microscopy. Journal of microscopy, 198(2):82-87, 2000. doi: 10.1046/j.1365-2818.2000.00710.x.

5. Stefan W Hell and Jan Wichmann. Breaking the diffraction resolution limit by stimulated emission: Stimulated-emission-depletion fluorescence microscopy. Optics letters, 19(11): 780-782, 1994. doi: 10.1364/ol.19.000780.

6. Helena Soares, Ricardo Henriques, Martin Sachse, Leandro Ventimiglia, Miguel A Alonso, Christophe Zimmer, Maria-Isabel Thoulouze, and Andrés Alcover. Regulated vesicle fusion generates signaling nanoterritories that control $\mathrm{T}$ cell activation at the immunological synapse. Journal of Experimental Medicine, 210(11):2415-2433, 2013. doi: 10.1084/jem.20130150.

7. Janine Scholefield, Ricardo Henriques, Anca F Savulescu, Elisabeth Fontan, Alix Boucharlat, Emmanuel Laplantine, Asma Smahi, Alain Israël, Fabrice Agou, and Musa M Mhlanga. Super-resolution microscopy reveals a preformed NEMO lattice structure that is collapsed in incontinentia pigmenti. Nature Communications, 7:12629, 2016. doi: $10.1038 /$ ncomms 12629 .

8. Joana G Silva, Nuno P Martins, Ricardo Henriques, and Helena Soares. HIV-1 Nef impairs the formation of calcium membrane territories controlling the signaling nanoarchitecture at the immunological synapse. The Journal of Immunology, 197(10):4042-4052, 2016. doi: 10.4049/jimmunol.1601132.

9. Robert DM Gray, David Albrecht, Corina Beerli, Moona Huttunen, Gary H Cohen, Ian J White, Jemima J Burden, Ricardo Henriques, and Jason Mercer. Nanoscale polarization of the entry fusion complex of vaccinia virus drives efficient fusion. Nature Microbiology, page 1, 2019. doi: 10.1038/s41564-019-0488-4.

10. Sina Waldchen, Julian Lehmann, Teresa Klein, Sebastian Van De Linde, and Markus Sauer. Light-induced cell damage in live-cell super-resolution microscopy. Scientific Reports, 5:1-12, 2015. doi: 10.1038/srep15348.

11. P Philippe Laissue, Rana A Alghamdi, Pavel Tomancak, Emmanuel G Reynaud, and Har Shroff. Assessing phototoxicity in live fluorescence imaging. Nature Methods, 14(7):657661, 2017. doi: 10.1038/nmeth.4344.

12. Jaroslav Icha, Michael Weber, Jennifer C. Waters, and Caren Norden. Phototoxicity in live fluorescence microscopy, and how to avoid it. BioEssays, 39(8):1-15, 2017. doi: 10.1002/bies.201700003.

13. Valentin Magidson and Alexey Khodjakov. Circumventing photodamage in live-cell microscopy. In Methods in Cell Biology, volume 114, pages 545-560. Elsevier, 2013.

14. Ram Dixit and Richard Cyr. Cell damage and reactive oxygen species production induced by fluorescence microscopy: Effect on mitosis and guidelines for non-invasive fluorescence microscopy. Plant Journal, 36(2):280-290, 2003. doi: 10.1046/j.1365-313x.2003.01868.x.

15. Katarina Logg, Kristofer Bodvard, Anders Blomberg, and Mikael Käll. Investigations on light-induced stress in fluorescence microscopy using nuclear localization of the transcription factor Msn2p as a reporter. FEMS Yeast Research, 9(6):875-884, 2009. doi: 10.1111/j.1567-1364.2009.00536.x.

16. Yoram Devary, Caridad Rosette, Joseph A. Didonato, and Michael Karin. NF- $\kappa$ B activation by ultraviolet light not dependent on a nuclear signal. Science, 261(5127):1442-1445, 1993. doi: $10.1126 /$ science. 8367725 .

17. Alnawaz Rehemtulla, Christin A Hamilton, Arul M Chinnaiyan, and Vishva M Dixit. Ultraviolet radiation-induced apoptosis is mediated by activation of CD-95 (Fas/APO-1). Journal of Biological Chemistry, 272(41):25783-25786, 1997. doi: 10.1074/jbc.272.41.25783.

18. MJ Peak and JG Peak. Single-strand breaks induced in Bacillus subtilis DNA by ultraviole light: Action spectrum and properties. Photochemistry and Photobiology, 35(5):675-680, 1982. doi: 10.1111/j.1751-1097.1982.tb02628.x.

19. Meyrick J. Peak, Jennifer G. Peak, and Bruce A. Carnes. Induction of Direct and Indirect Single-Strand Breaks in Human Cell Dna By Far- and Near-Ultraviolet Radiations: Action Spectrum and Mechanisms. Photochemistry and Photobiology, 45(3):381-387, 1987. doi: 10.1111/j.1751-1097.1987.tb05390.x.

20. Bo Durbeej and Leif $A$. Eriksson. Reaction mechanism of thymine dimer formation in DNA induced by UV light. Journal of Photochemistry and Photobiology A: Chemistry, 152(1-3): 95-101, 2002. doi: 10.1016/s1010-6030(02)00180-6.

21. Douglas E. Brash, Jeffrey A. Rudolph, Jeffrey A. Simon, Anthony Lin, Gregory J. Mckenna, Howard P. Baden, Alan J. Halperin, and Jan Pontén. A role for sunlight in skin cancer: UVinduced p53 mutations in squamous cell carcinoma. Proceedings of the National Academy of Sciences, 88(22):10124-10128, 1991. doi: 10.1073/pnas.88.22.10124.

22. H Vrieling, M L Van Rooijen, N A Groen, M Z Zdzienicka, J W Simons, P H Lohman, and A A van Zeeland. DNA strand specificity for UV-induced mutations in mammalian cells. Molecular and Cellular Biology, 9(3):1277-1283, 1989. doi: 10.1128/mcb.9.3.1277.

23. Michael L. Cunningham, Jennifer S. Johnson, Susan M. Giovanazzi, and Meyrick J. Peak. Photosensitized production of superoxide anion by monochromatic $(290-405 \mathrm{~nm})$ ultraviolet irradiation of NADH and NADPH coenzymes. Photochemistry and Photobiology, 42(2): 125-128, 1985. doi: 10.1111/j.1751-1097.1985.tb01549.x.

24. Maor Eichler, Ronit Lavi, Asher Shainberg, and Rachel Lubart. Flavins are source of visible-light-induced free radical formation in cells. Lasers in Surgery and Medicine, 37(4): 314-319, 2005. doi: 10.1002/lsm.20239.

25. Philip E. Hockberger, Timothy A. Skimina, Victoria E. Centonze, Colleen Lavin, Su Chu, Soheil Dadras, Janardan K. Reddy, and John G. White. Activation of flavin-containing oxidases underlies light-induced production of $\mathrm{H} 2 \mathrm{O} 2$ in mammalian cells. Proceedings of the National Academy of Sciences, 96(11):6255-6260, 1999. doi: 10.1073/pnas.96.11. 6255.

26. G. Ya Fraikin, M. G. Strakhovskaya, and A. B. Rubin. The role of membrane-bound porphyrin-type compound as endogenous sensitizer in photodynamic damage to yeas plasma membranes. Journal of Photochemistry and Photobiology B: Biology, 34(2-3): 129-135, 1996. doi: 10.1016/1011-1344(96)07287-9.

27. Fernanda Ricchelli. Photophysical properties of porphyrins in biological membranes. Journal of Photochemistry and Photobiology, B: Biology, 29(2-3):109-118, 1995. doi: 10.1016/1011-1344(95)07155-u.

28. Nicole Kilian, Alexander Goryaynov, Mark D. Lessard, Giles Hooker, Derek Toomre, James E. Rothman, and Joerg Bewersdorf. Assessing photodamage in live-cell STED microscopy. Nature Methods, 15(10):755-756, 2018. doi: 10.1038/s41592-018-0145-5.

29. Christopher S Foote. Definition of Type I and Type II Photosensitized Oxidation. Photo chemistry and Photobiology, 54(5):659, 1991. doi: 10.1111/j.1751-1097.1991.tb02071.x.

30. William C. Burhans and Nicholas H. Heintz. The cell cycle is a redox cycle: Linking phasespecific targets to cell fate. Free Radical Biology and Medicine, 47(9):1282-1293, 2009 doi: 10.1016/j.freeradbiomed.2009.05.026.

31. Ricardo Henriques, Caron Griffiths, E Hesper Rego, and Musa M Mhlanga. PALM and STORM: Unlocking live-cell super-resolution. Biopolymers, 95(5):322-331, 2011. doi: 
10.1002/bip.21586

32. Yoshiki Kuse, Kenjiro Ogawa, Kazuhiro Tsuruma, Masamitsu Shimazawa, and Hideak Hara. Damage of photoreceptor-derived cells in culture induced by light emitting diodederived blue light. Scientific Reports, 4:1-12, 2014. doi: 10.1038/srep05223.

33. Z. Schilling, E. Frank, V. Magidson, J. Wason, J. Lončarek, K. Boyer, J. Wen, and A. Khodjakov. Predictive-focus illumination for reducing photodamage in live-cell microscopy. Journal of Microscopy, 246(2):160-167, 2012. doi: 10.1111/j.1365-2818.2012.03605.x.

34. Stephen Douthwright and Greenfield Sluder. Live Cell Imaging: Assessing the Phototoxicity of 488 and $546 \mathrm{~nm}$ Light and Methods to Alleviate it. Journal of Cellular Physiology, 232(9):2461-2468, 2017. doi: 10.1002/jcp.25588.

35. Jean Yves Tinevez, Joe Dragavon, Lamya Baba-Aissa, Pascal Roux, Emmanuelle Perret, Astrid Canivet, Vincent Galy, and Spencer Shorte. A quantitative method for measuring phototoxicity of a live cell imaging microscope. In Methods in Enzymology, volume 506 , pages 291-309. Elsevier Inc., 1 edition, 2012. ISBN 978-0-12-391856-7. doi: 10.1016/ B978-0-12-391856-7.00039-1.

36. P. M. Carlton, J. Boulanger, C. Kervrann, J.-B. Sibarita, J. Salamero, S. Gordon-Messer, D. Bressan, J. E. Haber, S. Haase, L. Shao, L. Winoto, A. Matsuda, P. Kner, S. Uzawa, M. Gustafsson, Z. Kam, D. A. Agard, and J. W. Sedat. Fast live simultaneous multiwavelength four-dimensional optical microscopy. Proceedings of the National Academy of Sciences, 107(37):16016-16022, 2010. doi: 10.1073/pnas.1004037107.

37. Martin M Knight, Susan R Roberts, David A Lee, and Dan L Bader. Live cell imaging using confocal microscopy induces intracellular calcium transients and cell death. American Journal of Physiology-Cell Physiology, 284(4):C1083-C1089, 2003. doi: 10.1152/ajpcell. 00276.2002.

38. Yicong Wu, Alireza Ghitani, Ryan Christensen, Anthony Santella, Zhuo Du, Gary Rondeau, Zhirong Bao, Daniel Colón-Ramos, and Hari Shroff. Inverted selective plane illumination microscopy (iSPIM) enables coupled cell identity lineaging and neurodevelopmental imaging in Caenorhabditis elegans. Proceedings of the National Academy of Sciences, 108(43):17708-13, 2011. doi: 10.1073/pnas.1108494108.

39. H. Schnekenburger, P. Weber, M. Wagner, S. Schickinger, V. Richter, T. Bruns, W.S.L. Strauss, and R. Wittig. Light exposure and cell viability in fluorescence microscopy. Journal of Microscopy, 245(3):311-318, 2012. doi: 10.1111/j.1365-2818.2011.03576.x.

40. Firas Mubaid and Claire M. Brown. Less is More: Longer Exposure Times with Low Light Intensity is Less Photo-Toxic. Microscopy Today, 25(6):26-35, 2017. doi: 10.1017/ s1551929517000980.

41. Colton Boudreau, Tse Luen Wee, Yan Rung Duh, Melissa P. Couto, Kimya H. Ardakani, and Claire M. Brown. Excitation light dose engineering to reduce photo-bleaching and photo-toxicity. Scientific Reports, 6, 2016. doi: 10.1038/srep30892.

42. Yong Wu, Xundong Wu, Rong Lu, Jin Zhang, Ligia Toro, and Enrico Stefani. Resonan Scanning with Large Field of View Reduces Photobleaching and Enhances Fluorescence Yield in STED Microscopy. Scientific Reports, 5(September):1-12, 2015. doi: 10.1038/ srep14766.

43. Elias A. Halabi, Dorothea Pinotsi, and Pablo Rivera-Fuentes. Photoregulated fluxional fluorophores for live-cell super-resolution microscopy with no apparent photobleaching. Nature Communications, 10(1), 2019. doi: 10.1038/s41467-019-09217-7.

44. Ken Jacobson, Zenon Rajfur, Eric Vitriol, and Klaus Hahn. Chromophore-assisted laser inactivation in cell biology. Trends in Cell Biology, 18(9):443-450, 2008. doi: 10.1016/j.tcb. 2008.07.001.

45. John H. Stockley, Kimberley Evans, Moritz Matthey, Katrin Volbracht, Sylvia Agathou, Jana Mukanowa, Juan Burrone, and Ragnhildur T. Káradóttir. Surpassing light-induced cell damage in vitro with novel cell culture media. Scientific Reports, $7(1): 1-11$, 2017. doi: 10.1038/s41598-017-00829-x.

46. Jurek W. Dobrucki, Dorota Feret, and Anna Noatynska. Scattering of exciting light by live cells in fluorescence confocal imaging: Phototoxic effects and relevance for FRAP studies. Biophysical Journal, 93(5):1778-1786, 2007. doi: 10.1529/biophysj.106.096636.

47. Cora Roechlecke, Annette Schaller, Lilla Knels, and Richard H.W. Funk. The influence of sublethal blue light exposure on human RPE cells. Molecular Vision, 15(September): 1929-1938, 2009.

48. R A Hoebe, C H Van Oven, T W J Gadella, P B Dhonukshe, C J F Van Noorden, and E M M Manders. Controlled light-exposure microscopy reduces photobleaching and phototoxicity in fluorescence live-cell imaging. Nature Biotechnology, 25(2):249-253, 2007. doi: 10. $1038 / n b t 1278$

49. Jing Ge, David K. Wood, David M. Weingeist, Somsak Prasongtanakij, Panida Navasumrit, Mathuros Ruchirawat, and Bevin P. Engelward. Standard fluorescent imaging of live cells is highly genotoxic. Cytometry Part A, $83 \mathrm{~A}(6)$ :552-560, 2013. doi: 10.1002/cyto.a.22291.

50. Denise $\mathrm{H}$. Hawkins and Heidi Abrahamse. The role of laser fluence in cell viability, proliferation, and membrane integrity of wounded human skin fibroblasts following Helium-Neon laser irradiation. Lasers in Surgery and Medicine, 38(1):74-83, 2006. doi: $10.1002 / 1 \mathrm{sm} .20271$

51. Alison McDonald, John Harris, Debbi MacMillan, John Dempster, and Gail McConnell. Light-induced Câ2+ transients observed in widefield epi-fluorescence microscopy of excitable cells. Biomedical Optics Express, 3(6):1266, 2012. doi: 10.1364/boe.3.001266.

52. Helmut J. Koester, Dagmar Baur, Rainer Uhl, and Stefan W. Hell. Ca2+ fluorescence imaging with pico- and femtosecond two-photon excitation: Signal and photodamage. Biophysical Journal, 77(4):2226-2236, 1999. doi: 10.1016/s0006-3495(99)77063-3.

53. Alexander Hopt and Erwin Neher. Highly nonlinear photodamage in two-photon fluorescence microscopy. Biophysical Journal, 80(4):2029-2036, 2001. doi: 10.1016/ s0006-3495(01)76173-5.

54. Chien Hui Chuang, Anne E. Carpenter, Beata Fuchsova, Terezina Johnson, Primal de Lanerolle, and Andrew S. Belmont. Long-Range Directional Movement of an Interphase Chromosome Site. Current Biology, 16(8):825-831, 2006. doi: 10.1016/j.cub.2006.03.059.

55. Tetsuhiro Minamikawa, Absorn Sriratana, David A. Williams, David N. Bowser, John S. Hill, and Phillip Nagley. Chloromethyl-X-rosamine (MitoTracker Red) photosensitises mitochondria and induces apoptosis in intact human cells. Journal of Cell Science, 112(14): 2419-2430, 1999

56. R. A. Hoebe, H. T.M. Van Der Voort, J. Stap, C. J.F. Van Noorden, and E. M.M. Man- ders. Quantitative determination of the reduction of phototoxicity and photobleaching by controlled light exposure microscopy. Journal of Microscopy, 231(1):9-20, 2008. doi: 10.1111/j.1365-2818.2008.02009.x.

57. David Richmond, Anna Payne-Tobin Jost, Talley Lambert, Jennifer Waters, and Hunter Elliott. DeadNet: Identifying Phototoxicity from Label-free Microscopy Images of Cells using Deep ConvNets. arXiv preprint arXiv:1701.06109, pages 1-19, 2017.

58. Richard Cole. Live-cell imaging: The cell's perspective. Cell Adhesion and Migration, 8(5): 452-459, 2014. doi: 10.4161/cam.28348.

59. Giuseppe Vicidomini, Paolo Bianchini, and Alberto Diaspro. STED super-resolved microscopy. Nature Methods, 15(3):173-182, 2018. doi: 10.1038/nmeth.4593.

60. Ricardo Henriques and Musa M Mhlanga. PALM and STORM: What hides beyond the Rayleigh limit? Biotechnology Journal: Healthcare Nutrition Technology, 4(6):846-857, 2009. doi: 10.1002/biot.200900024.

61. Tyler J Chozinski, Lauren A Gagnon, and Joshua C Vaughan. Twinkle, twinkle little star: Photoswitchable fluorophores for super-resolution imaging. FEBS letters, 588(19):36033612, 2014. doi: 10.1016/j.febslet.2014.06.043.

62. Graham T Dempsey, Joshua C Vaughan, Kok Hao Chen, Mark Bates, and Xiaowei Zhuang. Evaluation of fluorophores for optimal performance in localization-based superresolution imaging. Nature Methods, 8(12):1027-1036, 2011. doi: 10.1038/nmeth.1768.

63. Jervis Vermal Thevathasan, Maurice Kahnwald, Konstanty Cieśliński, Philipp Hoess, Sudheer Kumar Peneti, Manuel Reitberger, Daniel Heid, Krishna Chaitanya Kasuba, Sarah Janice Hoerner, Yiming Li, et al. Nuclear pores as versatile reference standards for quantitative superresolution microscopy. BioRxiv, page 582668, 2019. doi: $10.1101 / 582668$

64. Sebastian van de Linde, Ivan Krstić, Thomas Prisner, Sören Doose, Mike Heilemann, and Markus Sauer. Photoinduced formation of reversible dye radicals and their impact on super-resolution imaging. Photochemical \& photobiological sciences : Official journal of the European Photochemistry Association and the European Society for Photobiology, 10 (4):499-506, 2011. doi: 10.1039/c0pp00317d

65. Nathan C. Shaner, Gerard G. Lambert, Andrew Chammas, Yuhui Ni, Paula J. Cranfill, Michelle A. Baird, Brittney R. Sell, John R. Allen, Richard N. Day, Maria Israelsson, Michael W. Davidson, and Jiwu Wang. A bright monomeric green fluorescent protein derived from Branchiostoma lanceolatum. Nature Methods, 10(5):407-409, 2013. doi: 10.1038/nmeth.2413.

66. Daphne S. Bindels, Lindsay Haarbosch, Laura Van Weeren, Marten Postma, Katrin E. Wiese, Marieke Mastop, Sylvain Aumonier, Guillaume Gotthard, Antoine Royant, Mark A. Hink, and Theodorus W.J. Gadella. MScarlet: A bright monomeric red fluorescent protein for cellular imaging. Nature Methods, 14(1):53-56, 2016. doi: 10.1038/nmeth.4074.

67. Anika Hense, Benedikt Prunsche, Peng Gao, Yuji Ishitsuka, Karin Nienhaus, and G. Ulrich Nienhaus. Monomeric Garnet, a far-red fluorescent protein for live-cell STED imaging. Scientific Reports, 5:1-10, 2015. doi: 10.1038/srep18006.

68. Mingshu Zhang, Hao Chang, Yongdeng Zhang, Junwei Yu, Lijie Wu, Wei Ji, Juanjuan Chen, Bei Liu, Jingze Lu, Yingfang Liu, Junlong Zhang, Pingyong Xu, and Tao Xu. Rational design of true monomeric and bright photoactivatable fluorescent proteins. Nature Methods, 9(7):727-729, 2012. doi: 10.1038/nmeth.2021.

69. Alyssa B Rosenbloom, Sang-Hyuk Lee, Milton To, Antony Lee, Jae Yen Shin, and Carlos Bustamante. Optimized two-color super resolution imaging of Drp1 during mitochondrial fission with a slow-switching Dronpa variant. Proceedings of the National Academy of Sciences, 111(36):13093-8, 2014. doi: 10.1073/pnas.1320044111.

70. Yoshiyuki Arai, Hiroki Takauchi, Yuhei Ogami, Satsuki Fujiwara, Masahiro Nakano, Tomoki Matsuda, and Takeharu Nagai. Spontaneously Blinking Fluorescent Protein for Simple Single Laser Super-Resolution Live Cell Imaging. ACS Chemical Biology, 13(8):19381943, 2018. doi: 10.1021/acschembio.8b00200.

71. William P. Dempsey, Lada Georgieva, Patrick M. Helbling, Ali Y. Sonay, Thai V. Truong, Michel Haffner, and Periklis Pantazis. In vivo single-cell labeling by confined primed conversion. Nature Methods, 12(7):645-648, 2015. doi: 10.1038/nmeth.3405.

72. Manuel Alexander Mohr and Periklis Pantazis. Primed Conversion: The New Kid on the Block for Photoconversion. Chemistry - A European Journal, 24(33):8268-8274, 2018. doi: 10.1002/chem.201705651.

73. Bartosz Turkowyd, Alexander Balinovic, David Virant, Haruko G.Gölz Carnero, Fabienne Caldana, Marc Endesfelder, Dominique Bourgeois, and Ulrike Endesfelder. A General Mechanism of Photoconversion of Green-to-Red Fluorescent Proteins Based on Blue and Infrared Light Reduces Phototoxicity in Live-Cell Single-Molecule Imaging. Angewandte Chemie - International Edition, 56(38):11634-11639, 2017. doi: 10.1002/anie.201702870.

74. Xi Zhang, Mingshu Zhang, Dong Li, Wenting He, Jianxin Peng, Eric Betzig, and Pingyong $\mathrm{Xu}$. Highly photostable, reversibly photoswitchable fluorescent protein with high contrast atio for live-cell superresolution microscopy. Proceedings of the National Academy of Sciences, 113(37):10364-10369, 2016. doi: 10.1073/pnas.1611038113.

75. Sheng Wang, Xuanze Chen, Lei Chang, Miao Ding, Ruiying Xue, Haifeng Duan, and Yujie Sun. GMars-T Enabling Multimodal Subdiffraction Structural and Functional Fluorescence Imaging in Live Cells. Analytical Chemistry, 90(11):6626-6634, 2018. doi: 10.1021/acs. analchem.8b00418

76. Maksim V Sednev, Christian A Wurm, Vladimir N Belov, and Stefan W Hell. Carborhodol: A new hybrid fluorophore obtained by combination of fluorescein and carbopyronine dye cores. Bioconjugate chemistry, 24(4):690-700, 2013. doi: 10.1021/bc3006732.

77. Jonathan B. Grimm, Anand K. Muthusamy, Yajie Liang, Timothy A. Brown, William C. Lemon, Ronak Patel, Rongwen Lu, John J. Macklin, Philipp J. Keller, Na Ji, and Luke D. avis. A general method to fine-tune fluorophores for live-cell and in vivo imaging. Nature Methods, 14(10):987-994, 2017. doi: 10.1038/nmeth.4403.

78. Pablo Mateos-Gil, Sebastian Letschert, Sören Doose, and Markus Sauer. SuperResolution Imaging of Plasma Membrane Proteins with Click Chemistry. Frontiers in Cell and Developmental Biology, 4(September):98, 2016. doi: 10.3389/fcell.2016.00098.

79. Alexander D. Thompson, Mitchell H. Omar, Felix Rivera-Molina, Zhiqun Xi, Anthony J. Koleske, Derek K. Toomre, and Alanna Schepartz. Long-Term Live-Cell STED Nanoscopy of Primary and Cultured Cells with the Plasma Membrane HIDE Probe Dil-SiR. Angewandte Chemie - International Edition, 56(35):10408-10412, 2017. doi: 10.1002/anie. 
201704783.

80. Grazvydas Lukinavicius, Luc Reymond, Elisa D'Este, Anastasiya Masharina, Fabian Göttfert, Haisen Ta, Angelika Güther, Mathias Fournier, Stefano Rizzo, Herbert Waldmann, Claudia Blaukopf, Christoph Sommer, Daniel W Gerlich, Hans-Dieter Arndt, Stefan W Hell, and Kai Johnsson. Fluorogenic probes for live-cell imaging of the cytoskeleton. Nature Methods, 11:731 EP -, 2014. doi: 10.1038/nmeth.2972.

81. Eszter Kozma and Péter Kele. Fluorogenic probes for super-resolution microscopy. Org Biomol. Chem., 17(2):215-233, 2019. doi: 10.1039/c8ob02711k.

82. S.-H. Shim, C. Xia, G. Zhong, H. P. Babcock, J. C. Vaughan, B. Huang, X. Wang, C. Xu, G. $\mathrm{Q}$. Bi, and $\mathrm{X}$. Zhuang. Super-resolution fluorescence imaging of organelles in live cells with photoswitchable membrane probes. Proceedings of the National Academy of Sciences, 109(35):13978-13983, 2012. doi: 10.1073/pnas.1201882109.

83. Mayeul Collot, Pichandi Ashokkumar, Halina Anton, Emmanuel Boutant, Orestis Faklaris, Thierry Galli, Yves Mély, Lydia Danglot, and Andrey S Klymchenko. MemBright: A Family of Fluorescent Membrane Probes for Advanced Cellular Imaging and Neuroscience. Cell Chemical Biology, 26(4):600 - 614.e7, 2019. doi: 10.1016/j.chembiol.2019.01.009.

84. Lu Wang, Michelle S Frei, Aleksandar Salim, and Kai Johnsson. Small-Molecule Fluorescent Probes for Live-Cell Super-Resolution Microscopy. Journal of the American Chemical Society, 141(7):2770-2781, 2019. doi: 10.1021/jacs.8b11134.

85. Luke D. Lavis. Teaching Old Dyes New Tricks: Biological Probes Built from Fluorescein and Rhodamines. Annual Review of Biochemistry, 86(1):825-843, 2017. doi: 10.1146/ annurev-biochem-061516-044839.

86. Arnaud Gautier, Alexandre Juillerat, Christian Heinis, Ivan Reis Corrêa, Maik Kindermann, Florent Beaufils, and Kai Johnsson. An Engineered Protein Tag for Multiprotein Labeling in Living Cells. Chemistry and Biology, 15(2):128-136, 2008. doi: 10.1016/j.chembiol.2008. 01.007.

87. Georgyi V Los, Lance P Encell, Mark G McDougall, Danette D Hartzell, Natasha Karassina, Chad Zimprich, Monika G Wood, Randy Learish, Rachel Friedman Ohana, Marjeta Urh, Dan Simpson, Jacqui Mendez, Kris Zimmerman, Paul Otto, Gediminas Vidugiris, Ji Zhu, Aldis Darzins, Dieter H Klaubert, Robert F Bulleit, and Keith V Wood. HaloTag: A novel protein labeling technology for cell imaging and protein analysis. ACS chemical biology, 3(6):373-82, 2008. doi: 10.1021/cb800025k.

88. B. Albert Griffin, Stephen R. Adams, and Roger Y. Tsien. Specific covalent labeling of recombinant protein molecules inside live cells. Science, 281(5374):269-72, 1998. doi: $10.1126 /$ science.281.5374.269.

89. Mickaël Lelek, Francesca Di Nunzio, Ricardo Henriques, Pierre Charneau, Nathalie Arhel, and Christophe Zimmer. Superresolution imaging of HIV in infected cells with FIAsHPALM. Proceedings of the National Academy of Sciences, 109(22):8564-9, 2012. doi: 10.1073/pnas.1013267109.

90. Till Stephan, Axel Roesch, Dietmar Riedel, and Stefan Jakobs. Live-cell STED microscopy of mitochondrial cristae. bioRxiv, 2019. doi: 10.1101/640920.

91. Shin Nosuke Uno, Dhermendra K. Tiwari, Mako Kamiya, Yoshiyuki Arai, Takeharu Nagai, and Yasuteru Urano. A guide to use photocontrollable fluorescent proteins and synthetic smart fluorophores for nanoscopy. Microscopy, 64(4):263-277, 2015. doi: 10.1093/jmicro/ dfv037.

92. Hideo Takakura, Yongdeng Zhang, Roman S. Erdmann, Alexander D. Thompson, Yu Lin, Brian McNellis, Felix Rivera-Molina, Shin Nosuke Uno, Mako Kamiya, Yasuteru Urano, James E. Rothman, Joerg Bewersdorf, Alanna Schepartz, and Derek Toomre. Long timelapse nanoscopy with spontaneously blinking membrane probes. Nature Biotechnology, 35(8):773-780, 2017. doi: 10.1038/nbt.3876.

93. Chenguang Wang, Masayasu Taki, Yoshikatsu Sato, Aiko Fukazawa, Tetsuya Higashiyama, and Shigehiro Yamaguchi. Super-Photostable Phosphole-Based Dye for Multiple-Acquisition Stimulated Emission Depletion Imaging. Journal of the American Chemical Society, 139(30):10374-10381, 2017. doi: 10.1021/jacs.7b04418.

94. Ling Chu, Jonathan Tyson, Juliana E Shaw, Felix Rivera-Molina, Anthony J Koleske, Alanna Schepartz, and Derek K Toomre. Two-color nanoscopy of organelles for extended times with HIDE probes. bioRxiv, 2019. doi: 10.1101/647065.

95. Alexey N. Butkevich, Vladimir N. Belov, Kirill Kolmakov, Viktor V. Sokolov, Heydar Shojaei, Sven C. Sidenstein, Dirk Kamin, Jessica Matthias, Rifka Vlijm, Johann Engelhardt, and Stefan W. Hell. Hydroxylated Fluorescent Dyes for Live-Cell Labeling: Synthesis, Spectra and Super-Resolution STED. Chemistry (Weinheim an der Bergstrasse, Germany), 23 (50):12114-12119, 2017. doi: 10.1002/chem.201701216.

96. Ivana Nikić, Gemma Estrada Girona, Jun Hee Kang, Giulia Paci, Sofya Mikhaleva, Christine Koehler, Nataliia V. Shymanska, Camilla Ventura Santos, Daniel Spitz, and Edward A. Lemke. Debugging Eukaryotic Genetic Code Expansion for Site-Specific Click-PAINT Super-Resolution Microscopy. Angewandte Chemie (International ed. in English), 55(52): 16172-16176, 2016. doi: 10.1002/anie.201608284.

97. Abdullah O Khan, Victoria A Simms, Jeremy A Pike, Steven G Thomas, and Neil V Morgan CRISPR-Cas9 Mediated Labelling Allows for Single Molecule Imaging and Resolution Scientific reports, 7(1):8450, 2017. doi: 10.1038/s41598-017-08493-x.

98. Tomer Schvartz, Noa Aloush, Inna Goliand, Inbar Segal, Dikla Nachmias, Eyal Arbely, and Natalie Elia. Direct fluorescent-dye labeling of $\alpha$-tubulin in mammalian cells for live cell and superresolution imaging. Molecular biology of the cell, 28(21):2747-2756, 2017. doi: 10.1091/mbc.e17-03-0161.

99. Michael Wagner, Petra Weber, Thomas Bruns, Wolfgang S.L. Strauss, Rainer Wittig, and Herbert Schneckenburger. Light dose is a limiting factor to maintain cell viability in fluorescence microscopy and single molecule detection. International Journal of Molecular Sciences, 11(3):956-966, 2010. doi: 10.3390/ijms11030956.

100. Maria E. Bulina, Konstantin A. Lukyanov, Olga V. Britanova, Daria Onichtchouk, Sergey Lukyanov, and Dmitriy M. Chudakov. Chromophore-assisted light inactivation (CALI) using the phototoxic fluorescent protein KillerRed. Nature Protocols, 1(2):947-953, 2006. doi: 10.1038 /nprot.2006.89.

101. Melanie M Frigault, Judith Lacoste, Jody L Swift, and Claire M Brown. Live-cell microscopy tips and tools. Journal of Cell Science, 122(Pt 6):753-67, 2009. doi: 10.1242/jcs.033837.

102. L. Song, E. J. Hennink, I. T. Young, and H. J. Tanke. Photobleaching kinetics of fluorescein in quantitative fluorescence microscopy. Biophysical Journal, 68(6):2588-2600, 1995. doi: 10.1016/s0006-3495(95)80442-x.

103. Jurek W Dobrucki. Interaction of oxygen-sensitive luminescent probes $\mathrm{Ru}$ (phen) $32+$ and Ru (bipy) $32+$ with animal and plant cells in vitro: Mechanism of phototoxicity and conditions for non-invasive oxygen measurements. Journal of Photochemistry and Photobiology B: Biology, 65(2-3):136-144, 2001. doi: 10.1016/s1011-1344(01)00257-3.

104. J. A. Bloom and W. W. Webb. Photodamage to intact erythrocyte membranes at high lase intensities: Methods of assay and suppression. Journal of Histochemistry and Cytochemistry, 32(6):608-616, 1984. doi: 10.1177/32.6.6725935.

105. Clare M. Waterman-Storer, Joseph W. Sanger, and Jean M. Sanger. Dynamics of organelles in the mitotic spindles of living cells: Membrane and microtubule interactions. Cell Motility and the Cytoskeleton, 26(1):19-39, 1993. doi: 10.1002/cm.970260104.

106. Wanqing Xiang, M. Julia Roberti, Jean Karim Hériché, Sébastien Huet, Stephanie Alexander, and Jan Ellenberg. Correlative live and super-resolution imaging reveals the dynamic structure of replication domains. Journal of Cell Biology, 217(6):1973-1984, 2018. doi: 10.1083/jcb.201709074.

107. Ron Kohen and Abraham Nyska. Oxidation of Biological Systems: Oxidative Stress Phenomena, Antioxidants, Redox Reactions, and Methods for Their Quantification. Toxicologic Pathology, 30(6):620-650, 2002. doi: 10.1080/01926230290166724.

108. T. W. Wu, N. Hashimoto, J. Wu, D. Carey, R. K. Li, D. A.G. Mickle, and R. D. Weisel. The cytoprotective effect of Trolox demonstrated with three types of human cells. Biochemistry and Cell Biology, 68(10):1189-1194, 1990. doi: 10.1139/090-176

109. Ivan Rasnik, Sean A. McKinney, and Taekjip Ha. Nonblinking and long-lasting singlemolecule fluorescence imaging. Nature Methods, 3(11):891-893, 2006. doi: 10.1038/ nmeth934.

110. Alexey M. Bogdanov, Elena I. Kudryavtseva, and Konstantin A. Lukyanov. Anti-Fading Media for Live Cell GFP Imaging. PLoS ONE, 7(12):1-4, 2012. doi: 10.1371/journal.pone. 0053004

111. Peter Atkins and Julio de Paula. Physical Chemistry. W.H. Freeman and Company, New York, US, eighth edition, 2006. ISBN 978-0-7167-8759-4. doi: 10.1007/SpringerReferencel $\lfloor 77053$.

112. Jerker Widengren, Andriy Chmyrov, Christian Eggeling, Per Åke Löfdahl, and Claus A.M. Seidel. Strategies to improve photostabilities in ultrasensitive fluorescence spectroscopy. Journal of Physical Chemistry A, 111(3):429-440, 2007. doi: 10.1021/jp0646325.

113. H Giloh and J W Sedat. Fluorescence microscopy: Reduced photobleaching of rhodamine and fluorescein protein conjugates by n-propyl gallate. Science, 217(4566):1252-5, 1982. doi: $10.1126 /$ science. 7112126 .

114. K. Valnes and P. Brandtzaeg. Retardation of immunofluorescence fading during microscopy. Journal of Histochemistry and Cytochemistry, 33(8):755-761, 1985 . doi: $10.1177 / 33.8 .3926864$

115. G. D. Johnson, R. S. Davidson, K. C. McNamee, G. Russell, D. Goodwin, and E. J. Holborow. Fading of immunofluorescence during microscopy: A study of the phenomenon and its remedy. Journal of Immunological Methods, 55(2):231-242, 1982. doi: 10.1016/0022-1759(82)90035-7.

116. A Longin, C Souchier, M Ffrench, and P A Bryon. Comparison of anti-fading agents used in fluorescence microscopy: Image analysis and laser confocal microscopy study. The journal of histochemistry and cytochemistry : official journal of the Histochemistry Society, 41(12):1833-40, 1993. doi: 10.1177/41.12.8245431.

117. A. N. Fletcher and M. E. Pietrak. Laser dye stability, part 10. Effects of DABCO on flashlamp pumping of coumarin dyes. Applied Physics B Photophysics and Laser Chemistry, 37(3):151-157, 1985. doi: 10.1007/bf00692078.

118. M. Ono, T. Murakami, A. Kudo, M. Isshiki, H. Sawada, and A. Segawa. Quantitative comparison of anti-fading mounting media for confocal laser scanning microscopy. Journal of Histochemistry and Cytochemistry, 49(3):305-311, 2001. doi: 10.1177/ 002215540104900304

119. Alexey M. Bogdanov, Ekaterina A. Bogdanova, Dmitriy M. Chudakov, Tatiana V. Gorodnicheva, Sergey Lukyanov, and Konstantin A. Lukyanov. Cell culture medium affects GFP photostability: A solution. Nature Methods, 6(12):859-860, 2009. doi: 10.1038/ nmeth1209-859.

120. A. M. Edwards, E. Silva, B. Jofré, M. I. Becker, and A. E. De loannes. Visible ligh effects on tumoral cells in a culture medium enriched with tryptophan and riboflavin Journal of Photochemistry and Photobiology, B: Biology, 24(3):179-186, 1994 . doi: 10.1016/1011-1344(94)07020-2.

121. Agnieszka Grzelak, Blaej Rychlik, and Grzegorz Bartosz. Light-dependent generation of reactive oxygen species in cell culture media. Free Radical Biology and Medicine, 30(12): 1418-1425, 2001. doi: 10.1016/s0891-5849(01)00545-7.

122. GT Spierenburg, FTJJ Oerlemans, JPRM Van Laarhoven, et al. Phototoxicity of N-2 Hydroxyethylpiperazine-N. Cancer research, 44:2253-2254, 1984.

123. J S Zigler, J L Lepe-Zuniga, B Vistica, and I Gery. Analysis of the cytotoxic effects of light-exposed HEPES-containing culture medium. In vitro cellular \& developmental biology journal of the Tissue Culture Association, 21(5):282-7, 1985. doi: 10.1007/bf02620943.

124. Pedro Almada, Siân Culley, and Ricardo Henriques. PALM and STORM: Into large fields and high-throughput microscopy with sCMOS detectors. Methods, 88:109-121, 2015. doi: 10.1016/j.ymeth.2015.06.004.

125. Sébastien Herbert, Helena Soares, Christophe Zimmer, and Ricardo Henriques. Singlemolecule localization super-resolution microscopy: Deeper and faster. Microscopy and microanalysis, 18(6):1419-1429, 2012. doi: 10.1017/s1431927612013347.

126. Pedro Almada, Pedro M Pereira, Siân Culley, Ghislaine Caillol, Fanny Boroni-Rueda, Christina L Dix, Guillaume Charras, Buzz Baum, Romain F Laine, Christophe Leterrier, et al. Automating multimodal microscopy with NanoJ-Fluidics. Nature communications, 10 (1):1223, 2019. doi: 10.1038/s41467-019-09231-9.

127. Gerald Donnert, Christian Eggeling, and Stefan W. Hell. Major signal increase in fluorescence microscopy through dark-state relaxation. Nature Methods, 4(1):81-86, 2007. doi: 10.1038/nmeth986.

128. K. König, T. W. Becker, P. Fischer, I. Riemann, and K.-J. Halbhuber. Pulse-length de pendence of cellular response to intense near-infrared laser pulses in multiphoton microscopes. Optics Letters, 24(2):113, 1999. doi: 10.1364/ol.24.000113. 
129. Kevin T. Takasaki, Jun B. Ding, and Bernardo L. Sabatini. Live-cell superresolution imaging by pulsed STED two-photon excitation microscopy. Biophysical Journal, 104(4):770-777, 2013. doi: 10.1016/j.bpj.2012.12.053.

130. Philipp Bethge, Ronan Chéreau, Elena Avignone, Giovanni Marsicano, and U. Valentin Nägerl. Two-photon excitation STED microscopy in two colors in acute brain slices. Biophysical Journal, 104(4):778-785, 2013. doi: 10.1016/j.bpj.2012.12.054.

131. Elke Schmidt and Martin Oheim. Two-photon imaging induces brain heating and calcium microdomain hyperactivity in cortical astrocytes. bioRxiv, page 321091, 2018. doi: 10. 1101/321091.

132. Benjamin Harke, Jan Keller, Chaitanya K. Ullal, Volker Westphal, Andreas Schönle, and Stefan W. Hell. Resolution scaling in STED microscopy. Optics Express, 16(6):4154, 2008. doi: 10.1364/oe.16.004154.

133. Giuseppe Vicidomini, Gael Moneron, Kyu Y Han, Volker Westphal, Haisen Ta, Matthias Reuss, Johann Engelhardt, Christian Eggeling, and Stefan W Hell. Sharper low-power STED nanoscopy by time gating. Nature Methods, 8(7):571-3, 2011. doi: 10.1038/nmeth. 1624.

134. Yicong Wu and Hari Shroff. Faster, sharper, and deeper: Structured illumination microscopy for biological imaging. Nature Methods, 15(12):1011-1019, 2018. doi: 10.1038/ s41592-018-0211-z.

135. Andrew G York, Sapun H Parekh, Damian Dalle Nogare, Robert S Fischer, Kelsey Temprine, Marina Mione, Ajay B Chitnis, Christian A Combs, and Hari Shroff. Resolution doubling in live, multicellular organisms via multifocal structured illumination microscopy. Nature Methods, 9(7):749-754, 2012. doi: 10.1038/nmeth.2025.

136. Ingo Gregor, Martin Spiecker, Roman Petrovsky, Jörg Großhans, Robert Ros, and Jörg Enderlein. Rapid nonlinear image scanning microscopy. Nature Methods, 14(11):10871089, 2017. doi: 10.1038/nmeth.4467.

137. E. N. Ward and R. Pal. Image scanning microscopy: An overview. Journal of Microscopy, 266(2):221-228, 2017. doi: 10.1111/jmi.12534.

138. Xiaoshuai Huang, Junchao Fan, Liuju Li, Haosen Liu, Runlong Wu, Yi Wu, Lisi Wei, Heng Mao, Amit Lal, Peng Xi, Liqiang Tang, Yunfeng Zhang, Yanmei Liu, Shan Tan, and Liangyi Chen. Fast, long-term, super-resolution imaging with Hessian structured illumination microscopy. Nature Biotechnology, 36(5):451-459, 2018. doi: 10.1038/nbt.4115.

139. Emmanuel G. Reynaud, Uroš Kržič, Klaus Greger, and Ernst H K Stelzer. Light sheetbased fluorescence microscopy: More dimensions, more photons, and less photodamage. HFSP Journal, 2(5):266-275, 2008. doi: 10.2976/1.2974980.

140. Emmanuel G. Reynaud, Jan Peychl, Jan Huisken, and Pavel Tomancak. Guide to lightsheet microscopy for adventurous biologists. Nature Methods, 12(1):30-34, 2014. doi: 10.1038/nmeth.3222

141. Rory M. Power and Jan Huisken. A guide to light-sheet fluorescence microscopy for multiscale imaging. Nature Methods, 14(4):360-373, 2017. doi: 10.1038/nmeth.4224.

142. Francesca Cella Zanacchi, Zeno Lavagnino, Michela Perrone Donnorso, Alessio Del Bue, Laura Furia, Mario Faretta, and Alberto Diaspro. Live-cell 3D super-resolution imaging in thick biological samples. Nature Methods, 8(12):1047-1050, 2011. doi: 10.1038/nmeth. 1744.

143. Francesca Cella Zanacchi, Zeno Lavagnino, Mario Faretta, Laura Furia, and Alberto Diaspro. Light-Sheet Confined Super-Resolution Using Two-Photon Photoactivation. PLoS ONE, 8(7), 2013. doi: 10.1371/journal.pone.0067667.

144. Jeongmin Kim, Michal Wojcik, Yuan Wang, Seonah Moon, Emilia A Zin, Nadia Marnani, Zachary L Newman, John G Flannery, Ke Xu, and Xiang Zhang. Oblique-plane singlemolecule localization microscopy for tissues and small intact animals. Nature Methods, pages 1-5, 2019. doi: 10.1038/s41592-019-0510-z.

145. Patrick Hoyer, Gustavo De Medeiros, Bálint Balázs, Nils Norlin, Christina Besir, Janina Hanne, Hans Georg Kräusslich, Johann Engelhardt, Steffen J. Sahl, Stefan W. Hell, and Lars Hufnagel. Breaking the diffraction limit of light-sheet fluorescence microscopy by RESOLFT. Proceedings of the National Academy of Sciences, 113(13):3442-3446, 2016. doi: 10.1073/pnas.1522292113.

146. Ying S. Hu, Quan Zhu, Keri Elkins, Kevin Tse, Yu Li, James A.J. Fitzpatrick, Inder M. Verma, and Hu Cang. Light-sheet Bayesian microscopy enables deepcell super-resolution imaging of heterochromatin in live human embryonic stem cells. Optical Nanoscopy, 2(1): 1-12, 2013. doi: 10.1186/2192-2853-2-7.

147. Thomas A. Planchon, Liang Gao, Daniel E. Milkie, Michael W. Davidson, James A. Galbraith, Catherine G. Galbraith, and Eric Betzig. Rapid three-dimensional isotropic imaging of living cells using Bessel beam plane illumination. Nature Methods, 8(5):417-423, 2011. doi: $10.1038 / n$ meth. 1586

148. Liang Gao, Lin Shao, Christopher D. Higgins, John S. Poulton, Mark Peifer, Michael W. Davidson, Xufeng Wu, Bob Goldstein, and Eric Betzig. Noninvasive imaging beyond the diffraction limit of 3D dynamics in thickly fluorescent specimens. Cell, 151(6):1370-1385, 2012. doi: 10.1016/j.cell.2012.10.008.

149. Bo Jui Chang, Victor Didier Perez Meza, and Ernst H.K. Stelzer. csiLSFM combines lightsheet fluorescence microscopy and coherent Structured illumination for a lateral resolution below $100 \mathrm{~nm}$. Proceedings of the National Academy of Sciences, 114(19):4869-4874, 2017. doi: 10.1073/pnas.1609278114.

150. B.-C. Chen, Wesley R. Legant, K. Wang, Lin Shao, Daniel E. Milkie, Michael W. Davidson, Chris Janetopoulos, Xufeng S. Wu, J. A. Hammer, Zhe Liu, Brian P. English, Yuko MimoriKiyosue, Daniel P. Romero, Alex T. Ritter, Jennifer Lippincott-Schwartz, Lillian Fritz-Laylin, R. Dyche Mullins, Diana M. Mitchell, J. N. Bembenek, A.-C. Reymann, R. Bohme, S. W. Grill, Jennifer T. Wang, Geraldine Seydoux, U. Serdar Tulu, Daniel P. Kiehart, and Eric Betzig. Lattice light-sheet microscopy: Imaging molecules to embryos at high spatiotemporal resolution. Science, 346(6208):1257998-1257998, 2014. doi: 10.1126/science.1257998.

151. Nadya Chakrova, Alicia Soler Canton, Christophe Danelon, Sjoerd Stallinga, and Bernd Rieger. Adaptive illumination reduces photobleaching in structured illumination microscopy. Biomedical Optics Express, 7(10):4263, 2016. doi: 10.1364/boe.7.004263.

152. Jes Dreier, Marco Castello, Giovanna Coceano, Rodrigo Cáceres, Julie Plastino, Giuseppe Vicidomini, and Ilaria Testa. Smart scanning for low-illumination and fast RESOLFT nanoscopy in vivo. Nature Communications, 10(1):1-11, 2019. doi: 10.1038/ s41467-019-08442-4.
153. Thorsten Staudt, Andreas Engler, Eva Rittweger, Benjamin Harke, Johann Engelhardt, and Stefan W. Hell. Far-field optical nanoscopy with reduced number of state transition cycles. Optics Express, 19(6):5644, 2011. doi: 10.1364/oe.19.005644.

154. Fabian Göttfert, Tino Pleiner, Jörn Heine, Volker Westphal, Dirk Görlich, Steffen J. Sahl, and Stefan W. Hell. Strong signal increase in STED fluorescence microscopy by imaging regions of subdiffraction extent. Proceedings of the National Academy of Sciences, 114 (9):2125-2130, 2017. doi: 10.1073/pnas.1621495114

155. Jörn Heine, Matthias Reuss, Benjamin Harke, Elisa D'Este, Steffen J Sahl, and Stefan W Hell. Adaptive-illumination STED nanoscopy. Proceedings of the National Academy of Sciences, 114(37):9797-9802, 2017. doi: 10.1073/pnas.1708304114.

156. Daniel Sage, Thanh-An Pham, Hazen Babcock, Tomas Lukes, Thomas Pengo, Jerry Chao, Ramraj Velmurugan, Alex Herbert, Anurag Agrawal, Silvia Colabrese, et al. Superresolution fight club: Assessment of $2 \mathrm{D}$ and 3D single-molecule localization microscopy software. Nature Methods, 16(5):387, 2019. doi: 10.1038/s41592-019-0364-4

157. Angélique Jimenez, Karoline Friedl, and Christophe Leterrier. About samples, giving examples: Optimized procedures for Single Molecule Localization Microscopy. Methods, 2019. doi: 10.1016/j.ymeth.2019.05.008.

158. Thomas Dertinger, Ryan Colyer, Gopal lyer, Shimon Weiss, and Jörg Enderlein. Fast, background-free, 3D super-resolution optical fluctuation imaging (SOFI). Proceedings of the National Academy of Sciences, 106(52):22287-22292, 2009. doi: 10.1073/pnas. 0907866106 .

159. Stefan Geissbuehler, Azat Sharipov, Aurélien Godinat, Noelia L. Bocchio, Patrick A. Sandoz, Anja Huss, Nickels A. Jensen, Stefan Jakobs, Jörg Enderlein, F. Gisou van der Goot, Elena A. Dubikovskaya, Theo Lasser, and Marcel Leutenegger. Live-cell multiplane threedimensional super-resolution optical fluctuation imaging. Nature Communications, 5(1): 5830, 2014. doi: $10.1038 /$ ncomms6830.

160. Susan Cox, Edward Rosten, James Monypenny, Tijana Jovanovic-Talisman, Dylan T Burnette, Jennifer Lippincott-Schwartz, Gareth E Jones, and Rainer Heintzmann. Bayesian localization microscopy reveals nanoscale podosome dynamics. Nature Methods, $9(2)$ : 195-200, 2012. doi: 10.1038/nmeth.1812.

161. Nils Gustafsson, Siân Culley, George Ashdown, Dylan M. Owen, Pedro Matos Pereira, and Ricardo Henriques. Fast live-cell conventional fluorophore nanoscopy with ImageJ through super-resolution radial fluctuations. Nature Communications, 7:1-9, 2016. doi: 10.1038/ncomms 12471 .

162. Romain F Laine, Kalina L Tosheva, Nils Gustafsson, Robert DM Gray, Pedro Almada, David Albrecht, Gabriel T Risa, Fredrik Hurtig, Ann-Christin Lindås, Buzz Baum, et al. NanoJ: A high-performance open-source super-resolution microscopy toolbox. Journal of Physics D: Applied Physics, 52(16):163001, 2019. doi: 10.1088/1361-6463/ab0261.

163. Siân Culley, Kalina L. Tosheva, Pedro Matos Pereira, and Ricardo Henriques. SRRF: Universal live-cell super-resolution microscopy. International Journal of Biochemistry and Cell Biology, 101(May):74-79, 2018. doi: 10.1016/j.biocel.2018.05.014.

164. Richard J. Marsh, Siân Culley, and Angus J. Bain. Low power super resolution fluorescence microscopy by lifetime modification and image reconstruction. Optics Express, 22 (10):12327, 2014. doi: 10.1364/oe.22.012327.

165. Lucas von Chamier, Romain F. Laine, and Ricardo Henriques. Artificial intelligence for microscopy: What you should know. Biochemical Society Transactions, 0(February): BST20180391, 2019. doi: 10.1042/bst20180391

166. Chinmay Belthangady and Loic A. Royer. Applications, promises, and pitfalls of deep learning for fluorescence image reconstruction. Nature Methods, 2019. doi: 10.1038/ s41592-019-0458-z.

167. Martin Weigert, Uwe Schmidt, Tobias Boothe, Andreas Müller, Alexandr Dibrov, Akanksha Jain, Benjamin Wilhelm, Deborah Schmidt, Coleman Broaddus, Siân Culley, Mauricio Rocha-Martins, Fabián Segovia-Miranda, Caren Norden, Ricardo Henriques, Marino Zerial, Michele Solimena, Jochen Rink, Pavel Tomancak, Loic Royer, Florian Jug, and Eugene W. Myers. Content-aware image restoration: Pushing the limits of fluorescence microscopy. Nature Methods, 15(12):1090-1097, 2018. doi: 10.1038/s41592-018-0216-7.

168. Wei Ouyang, Andrey Aristov, Mickaël Lelek, Xian Hao, and Christophe Zimmer. Deep learning massively accelerates super-resolution localization microscopy. Nature Biotechnology, 36(5):460-468, 2018. doi: 10.1038/nbt.4106.

169. Linjing Fang, Fred Monroe, Sammy Weiser Novak, Lyndsey Kirk, Cara Schiavon, Seungyoon B. Yu, Tong Zhang, Melissa Wu, Kyle Kastner, Yoshiyuki Kubota, Zhao Zhang, Gulcin Pekkurnaz, John Mendenhall, Kristen Harris, Jeremy Howard, and Uri Manor. Deep Learning-Based Point-Scanning Super-Resolution Imaging. bioRxiv, 2019. doi: $10.1101 / 740548$

170. Siân Culley, David Albrecht, Caron Jacobs, Pedro Matos Pereira, Christophe Leterrier, Jason Mercer, and Ricardo Henriques. Quantitative mapping and minimization of superresolution optical imaging artifacts. Nature Methods, 15(4):263, 2018. doi: 10.1038/nmeth. 4605 .

171. L Schermelleh, G Ball, J Demmerle, R Kaufmann, I Davis, and IM Dobbie. SIMcheck: A toolbox for successful super-resolution SIM imaging. Scientific Reports, pages 1-11, 2015. doi: 10.1038/srep15915. 